\title{
miR-22 has a potent anti-tumour role with therapeutic potential in acute myeloid leukaemia
}

\author{
Xi Jiang ${ }^{1,2}$, Chao Hu ${ }^{1,2,3}$, Stephen Arnovitz², Jason Bugno ${ }^{4}$, Miao Yu ${ }^{5}$, Zhixiang Zuo ${ }^{1,2,6}$, Ping Chen ${ }^{2}$, Hao Huang ${ }^{2}$, \\ Bryan Ulrich ${ }^{2}$, Sandeep Gurbuxani ${ }^{7}$, Hengyou Weng ${ }^{1,2}$, Jennifer Strong ${ }^{1}$, Yungui Wang ${ }^{1,2,3}$, Yuanyuan Li $^{2}$, \\ Justin Salat ${ }^{2}$, Shenglai Li ${ }^{2}$, Abdel G. Elkahloun ${ }^{8}$, Yang Yang ${ }^{4}$, Mary Beth Neilly ${ }^{2}$, Richard A. Larson ${ }^{2}$, \\ Michelle M. Le Beau², Tobias Herold ${ }^{9}$, Stefan K. Bohlander ${ }^{10}$, Paul P. Liu' ${ }^{8}$, Jiwang Zhang ${ }^{11}$, Zejuan Li ${ }^{2,12}$, \\ Chuan $\mathrm{He}^{5}$, Jie $\mathrm{Jin}^{3}$, Seungpyo Hong ${ }^{4,13}$ \& Jianjun Chen ${ }^{1,2}$
}

MicroRNAs are subject to precise regulation and have key roles in tumorigenesis. In contrast to the oncogenic role of miR-22 reported in myelodysplastic syndrome (MDS) and breast cancer, here we show that miR-22 is an essential anti-tumour gatekeeper in de novo acute myeloid leukaemia (AML) where it is significantly downregulated. Forced expression of miR-22 significantly suppresses leukaemic cell viability and growth in vitro, and substantially inhibits leukaemia development and maintenance in vivo. Mechanistically, miR-22 targets multiple oncogenes, including CRTC1, FLT3 and MYCBP, and thus represses the CREB and MYC pathways. The downregulation of miR-22 in AML is caused by TET1/GFI1/EZH2/SIN3Amediated epigenetic repression and/or DNA copy-number loss. Furthermore, nanoparticles carrying miR-22 oligos significantly inhibit leukaemia progression in vivo. Together, our study uncovers a TET1/GFI1/EZH2/SIN3A/miR-22/CREB-MYC signalling circuit and thereby provides insights into epigenetic/genetic mechanisms underlying the pathogenesis of $A M L$, and also highlights the clinical potential of miR-22-based AML therapy.

\footnotetext{
${ }^{1}$ Department of Cancer Biology, University of Cincinnati, Cincinnati, Ohio 45219, USA. ${ }^{2}$ Section of Hematology/Oncology, Department of Medicine, University of Chicago, Chicago, Illinois 60637, USA. ${ }^{3}$ Department of Hematology, The First Affiliated Hospital Zhejiang University, Hangzhou, 310003 Zhejiang, China. ${ }^{4}$ Department of Biopharmaceutical Sciences College of Pharmacy, The University of Illinois, Chicago, Illinois 60612 , USA. ${ }^{5}$ Department of Chemistry and Institute for Biophysical Dynamics, Howard Hughes Medical Institute, University of Chicago, Chicago, Illinois 60637, USA. ${ }^{6}$ State Key Laboratory of Oncology in South China, Collaborative Innovation Center for Cancer Medicine, Sun Yat-sen University Cancer Center, 510060 Guangzhou, China. ${ }^{7}$ Department of Pathology, University of Chicago, Chicago, Illinois 60637, USA. ${ }^{8}$ Division of Intramural Research, National Human Genome Research Institute, NIH, Bethesda, Maryland 20892, USA. ${ }^{9}$ Department of Internal Medicine 3, University Hospital Grosshadern, Ludwig-Maximilians-Universität, 81377 Munich, Germany. ${ }^{10}$ Department of Molecular Medicine and Pathology, University of Auckland, Auckland 1142, New Zealand. ${ }^{11}$ Oncology Institute, Cardinal Bernardin Cancer Center, Loyola University Medical Center, Maywood, Illinois 60153, USA. ${ }^{12}$ Department of Human Genetics, University of Chicago, Chicago, Illinois 60637, USA. ${ }^{13}$ Integrated Science and Engineering Division, Underwood International College, Yonsei University, Incheon 406-840, Korea. Correspondence and requests for materials should be addressed to J.C. (email: chen3jj@ucmail.uc.edu).
} 
A s one of the most common and fatal forms of hematopoietic malignancies, acute myeloid leukaemia (AML) is frequently associated with diverse chromosome translocations (for example $\mathrm{t}(11 \mathrm{q} 23) / M L L$-rearrangements, $\mathrm{t}(15 ; 17) / P M L-R A R A$ and $\mathrm{t}(8 ; 21) / A M L 1-E T O)$ and molecular abnormalities (for example, internal tandem duplications of FLT3 (FLT3-ITD) and mutations in nucleophosmin $\left.\left(N P M 1 c^{+}\right)\right)^{1}$. Despite intensive chemotherapies, the majority of patients with AML fail to survive longer than 5 years $^{2,3}$. Thus, development of effective therapeutic strategies based on a better understanding of the molecular mechanisms underlying the pathogenesis of AML is urgently needed.

MicroRNAs (miRNAs) are a class of small, non-coding RNAs that post-transcriptionally regulate gene expression ${ }^{4}$. Individual miRNAs may play distinct roles in cancers originating from different tissues or even from different lineages of hematopoietic cells $^{4}$. It is unclear whether a single miRNA can play distinct roles between malignancies originating from the same hematopoietic lineage, such as de novo AML and myelodysplastic syndrome (MDS). Although around $30 \%$ of MDS cases transform to AML, the genetic and epigenetic landscapes of MDS or MDS-derived AML are largely different from those of de novo $\mathrm{AML}^{5,6}$. MDS and MDS-derived AML are more responsive to hypomethylating agents than de novo $\mathrm{AML}^{7}$. The molecular mechanisms underlying the distinct pathogenesis and drug response between MDS (or MDS-derived AML) and de novo AML remain unclear.

The ten-eleven translocation (Tet1/2/3) proteins play critical transcriptional regulatory roles in normal developmental processes as activators or repressors ${ }^{8-10}$. In contrast to the frequent loss-offunction mutations and tumour-suppressor role of TET2 observed in hematopoietic malignancies ${ }^{11-13}$, we recently reported that TET1 plays an essential oncogenic role in $M L L$-rearranged AML where it activates expression of homeobox genes ${ }^{14}$. However, it is unknown whether TET1 can also function as a transcriptional repressor in cancer. Moreover, Tet1-mediated regulation of miRNA expression has rarely been studied ${ }^{10}$.

In the present study, we demonstrate that miR-22, an oncogenic miRNA reported in breast cancer and $\mathrm{MDS}^{15,16}$, is significantly downregulated in most cases of de novo AML due to TET1/GFI1/EZH2/SIN3A-mediated epigenetic repression and/or DNA copy-number loss. miR-22 functions as an essential antitumour gatekeeper in various AML and holds great therapeutic potential to treat AML.

\section{Results}

The downregulation of miR-22 in de novo AML. Through Exiqon miRNA array profiling, we previously identified a set of miRNAs, such as miR-150, miR-148a, miR-29a, miR-29b, miR-184, miR-342, miR-423 and miR-22, which are significantly downregulated in AML compared with normal controls ${ }^{19}$. Here we showed that among all the above miRNAs, miR-150 and especially miR-22 exhibited the most significant and consistent inhibitory effect on $M L L$-AF9-induced cell immortalization in colonyforming/replating assays (CFA) (Supplementary Fig. 1a). In contrast to the reported upregulation of miR-22 in MDS ${ }^{16}$, our original microarray data ${ }^{17}$ (Fig. 1a,b) and new quantitative PCRindependent validation data (Supplementary Fig. 1b) demonstrated a significant and global downregulation of miR-22 in de novo AML relative to normal controls. Notably, miR-22 is significantly downregulated in AML samples $(P<0.05)$ compared with all three sub-populations of normal control cells, that is, normal $\mathrm{CD}_{3}{ }^{+}$hematopoietic stem/progenitor cells (HSPCs), CD33 ${ }^{+}$ myeloid progenitor cells, or mononuclear cells (MNCs) (Fig. 1a). Expression of miR-22 is significantly downregulated in all or the majority of individual subsets of AML samples than in the normal $\mathrm{CD}_{3}{ }^{+}$or $\mathrm{CD}_{3}{ }^{+}$cell samples (Fig. 1b).
To rule out the possibility that the inhibitory effect of miR-22 shown in Supplementary Fig. la was due to a non-specific effect of our miR-22 construct, we included the MSCV-PIG-miR-22 construct from Song et al. ${ }^{16}$ in a repeated CFA. Both miR-22 constructs dramatically inhibited $M L L-A F 9$-induced colony formation (Fig. 1c). As the 'seed' sequences at the $5^{\prime}$ end of individual miRNAs are essential for the miRNA-target binding ${ }^{18}$, we also mutated the 6-bases 'seed' sequence of miR-22 and found that the miR-22 mutant did not inhibit colony formation anymore (Fig. 1c). In human AML cells, forced expression of miR-22, but not miR-22 mutant, significantly inhibited cell viability and growth/proliferation, while promoting apoptosis (Supplementary Fig. 1c,d).

Furthermore, as miR-22 is globally downregulated in all major types of AML (Fig. 1b), we also investigated the role of miR-22 in colony formation induced by other oncogenic fusion genes, including $M L L-A F 10 / \mathrm{t}(10 ; 11), P M L-R A R A / \mathrm{t}(15 ; 17)$ and $A M L 1-$ $E T O 9 a / t(8 ; 21)$ (ref. 19). As expected, forced expression of miR-22 significantly inhibited colony formation induced by all individual oncogenic fusions; conversely, miR-22 knockout ${ }^{20}$ significantly enhanced colony forming (Fig. 1d). These results suggest that miR-22 likely plays a broad anti-tumour role in AML.

In accordance with the potential anti-tumour function of miR-22 in AML, miR-22 was expressed at a significantly higher level $(P<0.05)$ in human normal $\mathrm{CD}_{3}{ }^{+}$myeloid progenitor cells than in more immature CD34 ${ }^{+}$HSPCs or MNC cells (a mixed population containing both primitive progenitors and committed cells) (Fig. 1a,b), implying that miR-22 is upregulated during normal myelopoiesis. Similarly, we showed that miR-22 was also expressed at a significantly higher level in mouse normal bone marrow (BM) myeloid $\left(\mathrm{Gr}-1^{+} / \mathrm{Mac}-1^{+}\right)$cells, relative to lineage negative $\left(\mathrm{Lin}^{-}\right)$progenitor cells, long-term hematopoietic stem cells (LT-HSCs), short-term HSCs (ST-HSCs), and committed progenitors (CPs) (Supplementary Fig. 1e), further suggesting that miR-22 is upregulated in normal myelopoiesis.

The anti-tumour effect of miR-22 in the pathogenesis of AML. Through bone marrow transplantation (BMT) assays, we showed that forced expression of miR-22 (but not miR-22 mutant) dramatically blocked MLL-AF9 (MA9)-mediated leukemogenesis in primary BMT recipient mice, with a more potent inhibitory effect than miR-150 (Fig. 1e; Supplementary Fig. 2a). All MA9+ miR-22 mice exhibited normal morphologies in peripheral blood (PB), BM, spleen and liver tissues (Fig. 1f), with a substantially reduced c-Kit ${ }^{+}$ blast cell population in BM (Supplementary Fig. 2b). Forced expression of miR-22 also almost completely inhibited leukemogenesis induced by $M L L-A F 10$ (Fig. 1g; Supplementary Fig. 2a). Conversely, miR-22 knockout significantly promoted AML1-ETO9a (AE9a)-induced AML (Fig. 1h). Thus, the repression of miR-22 is critical for the development of primary AML. Notably, forced expression of miR-22 in MLL-AF9 and MLL-AF10 leukaemia mouse models caused only a 2-3-fold increase in miR-22 expression level (Supplementary Fig. 2a), in a degree comparable to the difference in miR-22 expression levels between human AML samples and normal controls (Fig. 1a), suggesting that a 2-3-fold change in miR-22 expression level appears to be able to exert significant physiological or pathological effects.

To examine whether the maintenance of AML is also dependent on the repression of miR-22, we performed secondary BMT assays. Forced expression of miR-22 remarkably inhibited progression of MLL-AF9-, AE9a- or FLT3-ITD/NPM1c ${ }^{+}$. induced AML in secondary recipient mice (Fig. 2a-d), resulting in largely normal morphologies in $\mathrm{PB}, \mathrm{BM}$, spleen and liver tissues (Fig. 2b; Supplementary Fig. 2c). Collectively, our findings demonstrate that miR-22 is a pivotal anti-tumour gatekeeper in both development and maintenance of various AML. 
Identification of critical target genes of miR-22 in AML. To identify potential targets of miR-22 in AML, we performed a series of data analysis. Analysis of In-house_81S (ref. 21) and TCGA_177S (ref. 22) data sets revealed a total of 999 genes exhibiting significant inverse correlations with miR-22 in expression. Of them, 137 genes, including 21 potential targets of miR-22 as predicted by TargetScan ${ }^{18}$ (Supplementary Table 1), were significantly upregulated in both human and mouse AML compared with normal controls as detected in two additional inhouse data sets ${ }^{14,23}$. Among the 21 potential targets, CRTC1,

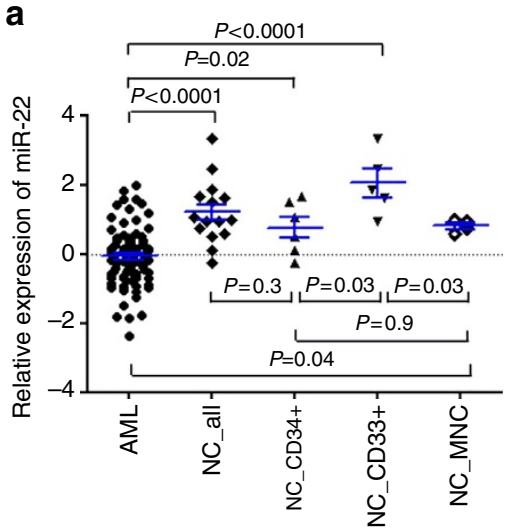

C

miR-22 ...GCCGCAGTAGTTCTTCAGTGGCAAGCTTTATGTCCTG. miR-22mut...GCCGCAGTAAGGTCCCAGTGGCAAGCTTTATGTCCTG.

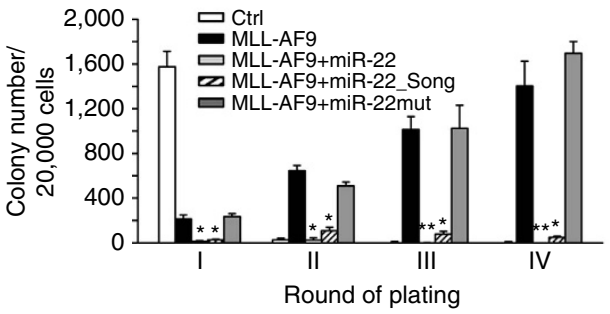

b

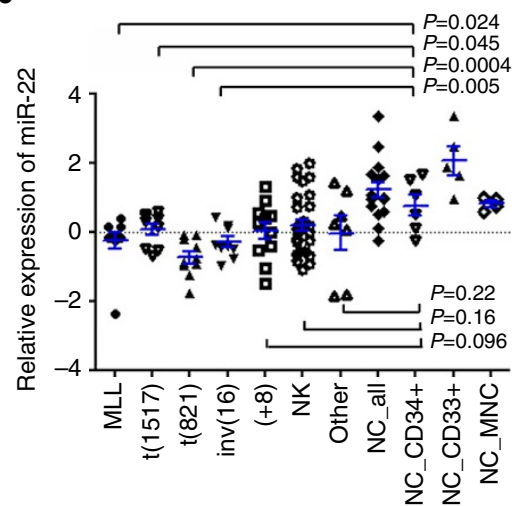

d

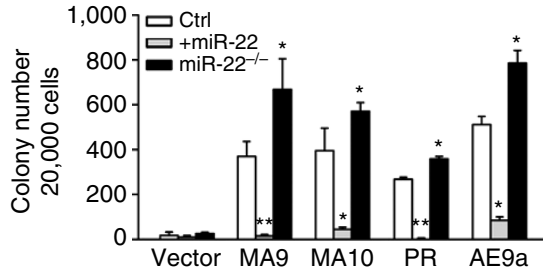

f

PB

SP

Liver

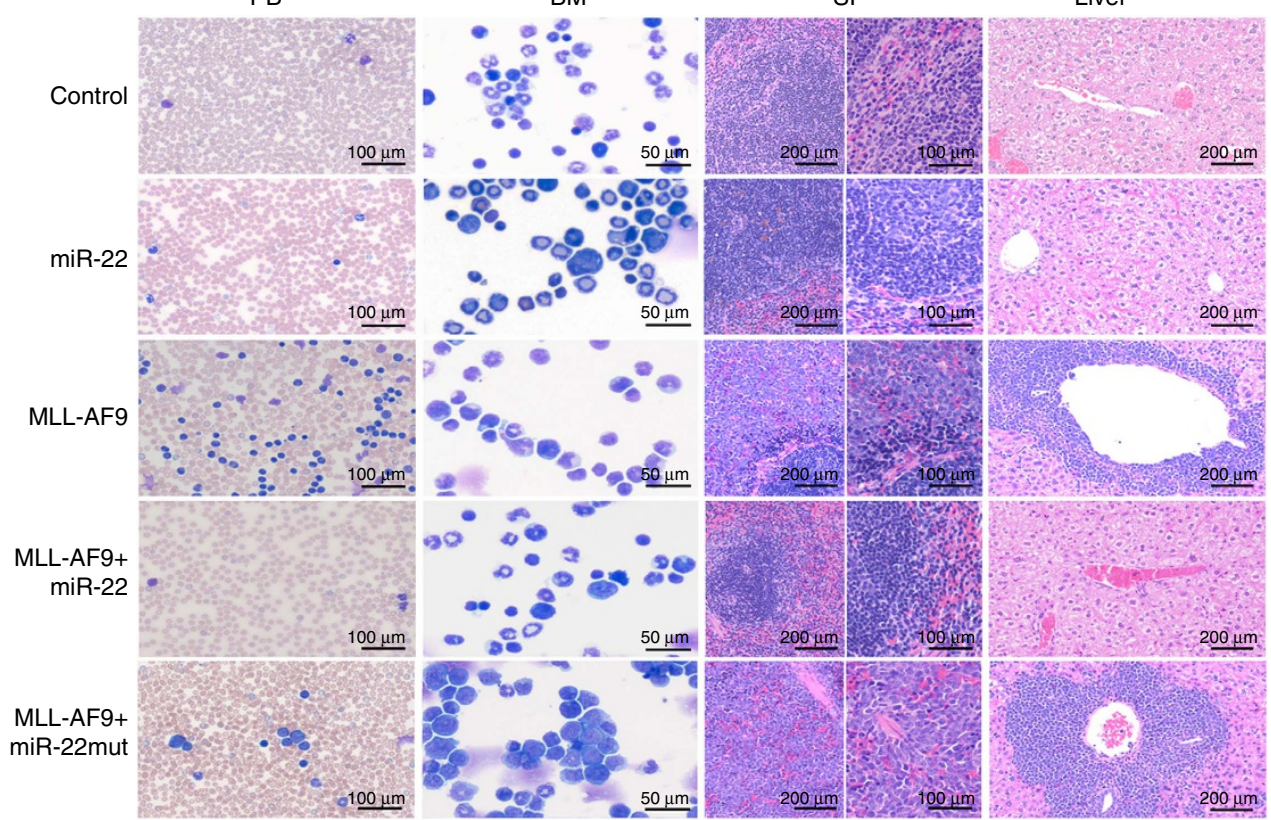

g

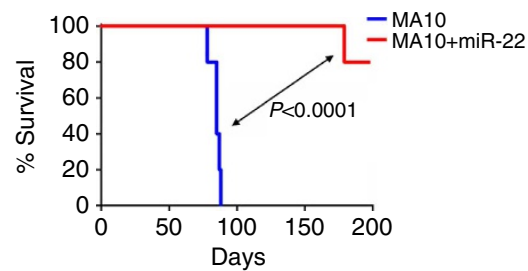

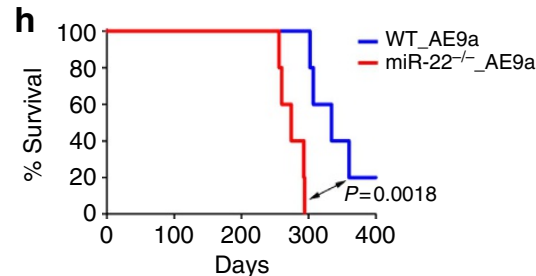


ETV6 and FLT3 are known oncogenes ${ }^{24-29}$. We then focused on these three genes, along with $M Y C B P$ that encodes the MYC-binding protein and is an experimentally validated target of miR-22 (ref. 30) although due to a technical issue it was not shown in the 21-gene list (Supplementary Table 1), for further studies.

As expected, all four genes were significantly downregulated in expression by ectopic expression of miR-22 in human MONOMAC-6/t(9;11) cells (Fig. 3a). The coincidence of downregulation of those genes and upregulation of miR-22 was also observed in mouse $M L L-E N L$-ERtm cells, a leukaemic cell line with an inducible $M L L-E N L$ derivative ${ }^{31}$, when MLL-ENL was depleted by 4-hydroxy-tamoxifen (4-OHT) withdrawal (Fig. 3b; Supplementary Fig. 3a). While MLL-AF9 remarkably promoted expression of those four genes in mouse BM progenitor cells, coexpressed miR-22 reversed the upregulation (Fig. 3c). In leukaemia BM blast cells of mice with $M L L-A F 9$-induced AML, the expression of Crtc1, Flt3 and Mycbp, but not Etv6, was significantly downregulated by co-expressed miR-22 (but not by miR-22 mutant) (Fig. 3d). Because miR-22-mediated downregulation of Etv 6 could be observed only in the in vitro models (Fig. $3 \mathrm{a}-\mathrm{c}$ ), but not in the in vivo model (Fig. $3 \mathrm{~d}$ ), which was probably due to the difference between in vitro and in vivo microenvironments, we decided to focus on the three target genes (that is, Crtc1, Flt3 and Mycbp) that showed consistent patterns between in vitro and in vivo for further studies. The repression of Crtc1, Flt3 and Mycbp was also found in leukaemia BM cells of mice with $A E 9 a$ or FLT3-ITD/NPM1c ${ }^{+}$-induced AML (Fig. 3e,f). As $M y c b p$ is already a known target of miR-22 (ref. 30), here we further confirmed that FLT3 and CRTC1 are also direct targets of miR-22 (Fig. 3g,h). The downregulation of CRTC1, FLT3 and MYCBP by miR-22 at the protein level was confirmed in both human and mouse leukaemic cells (Supplementary Fig. 3b,c). Overexpression of miR-22 had no significant influence on the level of leukaemia fusion genes (Supplementary Fig. 3d).

Co-expression of the coding region (CDS) of each of the three target genes (that is, CRTC1, FLT3 and MYCBP) largely reversed the effects of miR-22 on cell viability, apoptosis and proliferation (Fig. 4a-e). More importantly, in vivo BMT assays showed that co-expressing CRTC1, FLT3 or MYCBP largely rescued the inhibitory effect of miR-22 on leukemogenesis (Fig. 4f,g; Supplementary Fig. 3e). Our data thus suggest that CRTC1, FLT3 and MYCBP are functionally important targets of miR-22 in AML.
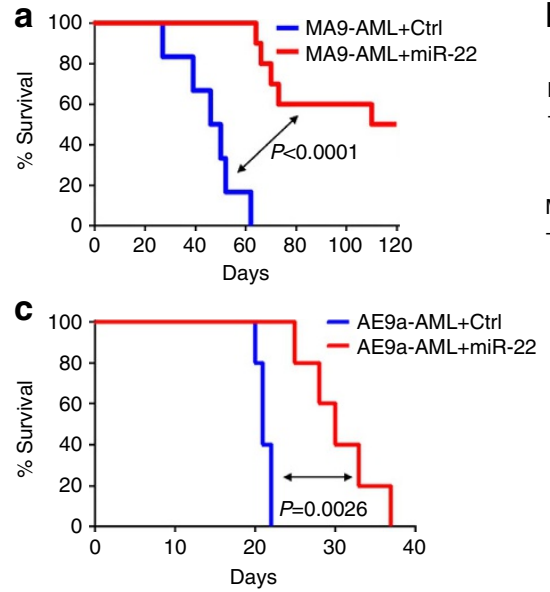

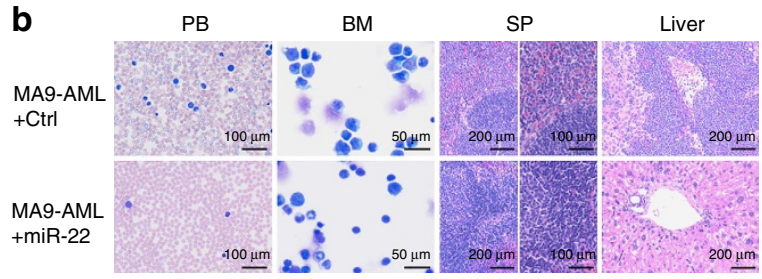

d

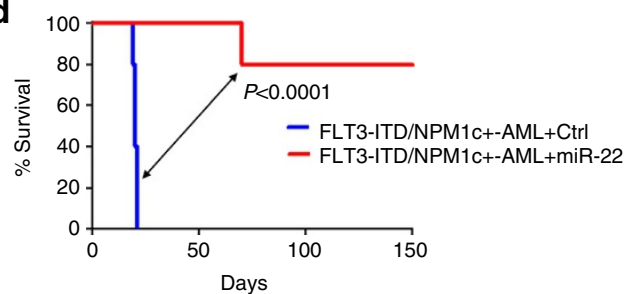

Figure 2 | Effect of miR-22 on the maintenance of AML in vivo. (a,b) Effect of miR-22 on the maintenance of MLL-AF9-induced AML in secondary BMT recipient mice. The secondary BMT recipients were transplanted with BM blast cells from the primary MLL-AF9 AML mice retrovirally transduced with MSCV-PIG + MSCVneo (MA9-AML + Ctrl; $n=7$ ) or MSCV-PIG + MSCVneo-miR-22 (MA9-AML + miR-22; $n=10$ ). Kaplan-Meier curves (a) and WrightGiemsa or H\&E-stained PB, BM, spleen and liver (b) of the secondary leukaemic mice are shown. (c,d) Effect of miR-22 on the maintenance/progression of AML1-ETO9a (AE9a)-induced AML (c) or FLT3-ITD/NPM1c ${ }^{+}$-induced AML (d) in secondary BMT recipient mice ( $n=5$ for each group). Kaplan-Meier curves and $P$ values (log-rank test) are shown.

Figure 1 | miR-22 inhibits AML cell transformation and leukemogenesis. (a,b) Exiqon microRNA profiling assay showed that miR-22 is significantly $(P<0.05)$ downregulated in the entire set of AML set $(n=85)$ (a) or each individual subset $(\mathbf{b})$, relative to normal controls. The expression data were $\log (2)$ transformed and mean-centred. Mean \pm s.e.m. values were shown. (c) Comparison of effects of in-house miR-22, miR-22_Song ${ }^{16}$ and miR-22 mutant (miR-22mut; see the mutation sequence at the top) on MLL-AF9-induced colony forming. CFAs were performed using mouse BM progenitor (Lin ${ }^{-}$) cells transduced with MSCV-neo + MSCV-PIG (Ctrl), MSCV-neo-MLL-AF9 + MSCV-PIG (MLL-AF9), or MSCV-neo-MLL-AF9 + MSCV-PIG-miR-22/miR22_Song/miR-22mut. (d) Effects of miR-22 on the colony forming induced by multiple fusion genes. CFA was performed using wild-type BM progenitor cells co-transduced with MSCV-neo-MLL-AF9 (MA9), -MLL-AF10 (MA10), -PML-RARA (PR) or -AML1-ETO9a (AE9a) ${ }^{19}$, together with MSCV-PIG (Ctrl) or MSCV-PIG-miR-22 ( + miR-22), as well as miR-22-/- BM progenitors co-transduced with individual fusion genes and MSCV-PIG. Colony counts (mean \pm s.d.) of the second round of plating are shown. ${ }^{\star} P<0.05 ;{ }^{* *} P<0.01$. (e,f) Effect of miR-22 on MLL-AF9-induced primary leukemogenesis. KaplanMeier curves are shown for six cohorts of transplanted mice including MSCVneo + MSCV-PIG (Ctrl; $n=5$ ), MSCVneo + MSCV-PIG-miR-22 (miR-22; $n=5)$, MSCVneo-MLL-AF9 + MSCV-PIG (MA9; $n=8)$, MSCVneo-MLL-AF9+MSCV-PIG-miR-150 (MA9 + miR-150, $n=6$ ), MSCVneo-MLL-AF9+ MSCVPIG-miR-22 (MA9 + miR-22; $n=10)$ and MSCVneo-MLL-AF9 + MSCV-PIG-miR-22mutant (MA9 + miR-22mut; $n=5)(\mathbf{e}) ;$ Wright-Giemsa stained PB and bone marrow (BM), and hematoxylin and eosin (H\&E) stained spleen and liver of the primary BMT recipient mice at the end point are shown (f). (g) Effect of miR-22 on MLL-AF10-induced primary leukemogenesis. Kaplan-Meier curves are shown for two cohorts of transplanted mice including MSCVneo-MLLAF10 + MSCV-PIG (MA10; $n=5$ ) and MSCVneo-MLL-AF10 + MSCV-PIG-miR-22 (MA10 + miR-22; $n=5$ ). (h) miR-22 knockout promotes AE9a-induced leukemogenesis. Kaplan-Meier curves are shown for mice transplanted with wild-type or miR-22-/- BM progenitor cells transduced MSCV-PIG-AE9a ( $n=5$ for each group). The $P$ values were generated by $t$-test $(\mathbf{a}-\mathbf{d})$ or log-rank test $(\mathbf{e}, \mathbf{g}, \mathbf{h})$. 
a

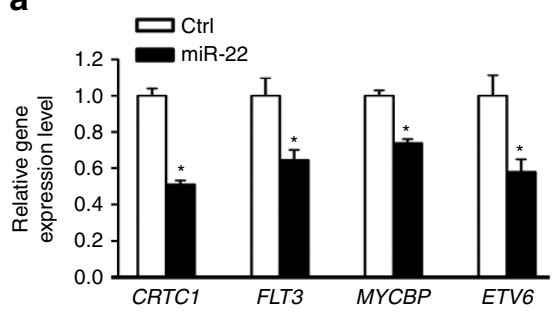

C

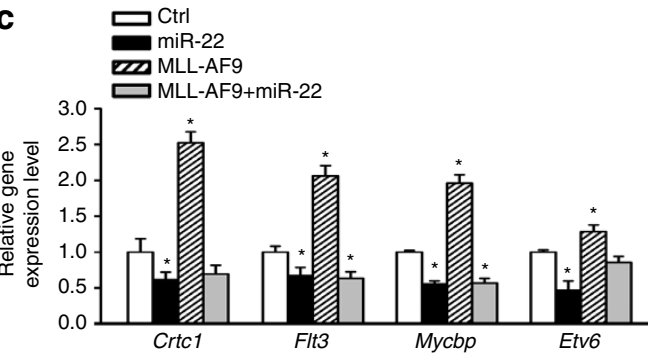

e

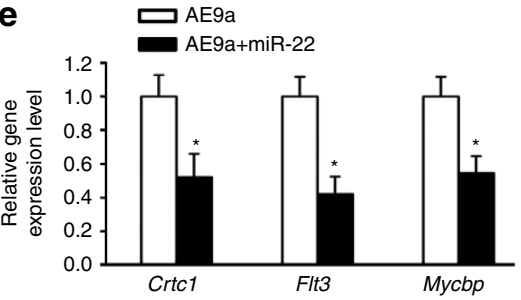

g

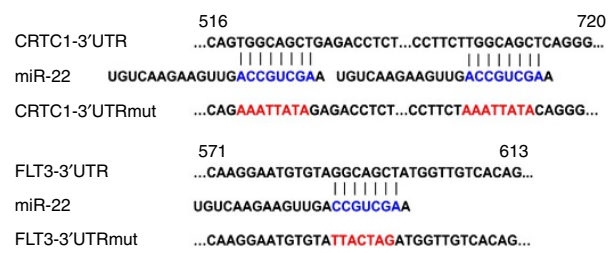

b

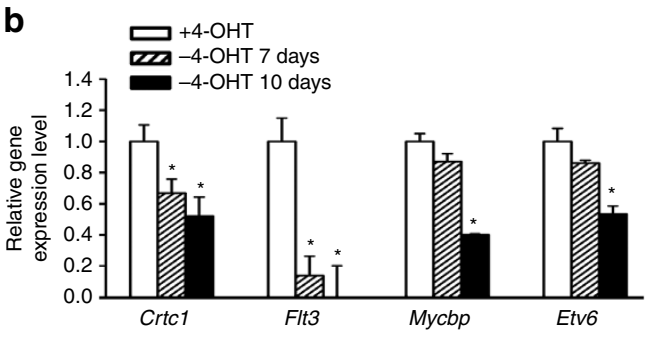

d

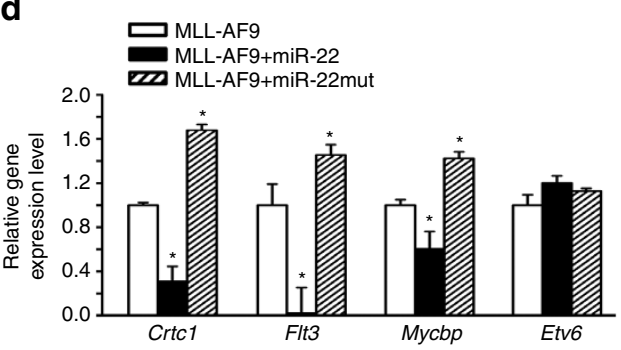

$\mathbf{f}$

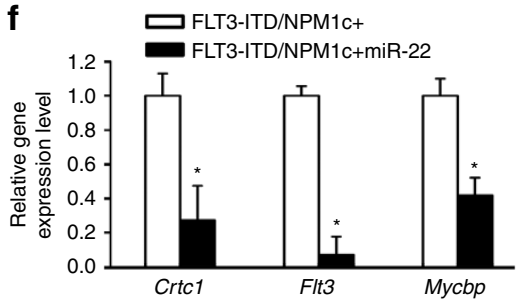

h

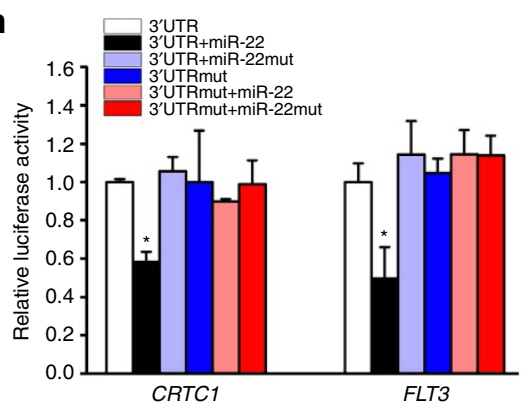

Figure 3 | miR-22 targets multiple oncogenes. (a) Downregulation of CRTC1, FLT3, MYCBP and ETV6 by forced expression of miR-22 in MONOMAC-6 cells. Expression of these genes was detected $48 \mathrm{~h}$ post transfection of MSCV-PIG (Ctrl) or MSCV-PIG-miR-22 (miR-22). (b) Crtc1, Flt3, Mycbp and Etv6 levels in MLL-ENL-ERtm cells after withdrawal of 4-OHT for 0, 7 or 10 days. (c) Expression levels of Crtc1, Flt3, Mycbp and Etv6 in mouse BM progenitor cells retrovirally transduced with MSCV-PIG + MSCV-neo (Ctrl), MSCV-PIG-miR-22 + MSCV-neo (miR-22), MSCV-PIG + MSCV-neo-MLL-AF9 (MLL-AF9) or MSCV-PIG-miR$22+$ MSCV-neo-MLL-AF9 (MLL-AF9 + miR-22). (d) Expression levels of Crtc1, Flt3, Mycbp and Etv6 in BM blast cells of leukaemic mice transplanted with MLL-AF9, MLL-AF9 + miR-22 or MLL-AF9 + miR-22mut primary leukaemic cells. (e,f) Expression levels of Crtc1, Flt3 and Mycbp in BM blast cells of leukaemic mice transplanted with MSCV-PIG or MSCV-PIG-miR-22-retrovirally transduced AE9a (e) or FLT3-ITD/NPM1c ${ }^{+}$(f) primary leukaemic cells. (g) Putative miR-22 target sites and mutants in the $3^{\prime}$ UTRs of CRTC1 (upper panel) and FLT3 (lower panel). (h) Effects of miR-22 on luciferase activity of the reporter gene bearing wild type or mutant 3'UTRs of CRTC1 or FLT3 in HEK293T cells. The mean \pm s.d. values from three replicates are shown. ${ }^{\star} P<0.05, t$-test.

miR-22 represses both $\mathrm{CREB}$ and MYC signalling pathways. CRTC1, a CREB-regulated transcription coactivator, facilitates CREB in regulating transcription of its targets, in both normal and malignant hematopoiesis ${ }^{24-26}$. CDK6, HOXA7 and RGS2 are known direct targets of CREB that are either positively (CDK6 and HOXA7) or negatively (RGS2) regulated by $\mathrm{CREB}^{32-35}$. In both In-house_81S (ref. 21) and TCGA_177S (ref. 22) data sets, CDK6 and HOXA7 inversely, while RGS2 positively, correlated with miR-22 in expression (Supplementary Table 2; Supplementary Fig. 3f). In leukaemic BM blast cells from primary and secondary BMT recipients, overexpression of miR-22 (but not miR-22 mutant) significantly downregulated expression of Cdk6 and Hoxa7, while upregulating Rgs2, which could be reversed by co-expressing CRTC1 (Supplementary Fig. 3g,h).
These results suggest that miR-22 represses the CREB signalling pathway in AML by targeting CRTC1.

MYCBP, a MYC-binding protein, is essential for MYC-mediated gene regulation ${ }^{30}$. FLT3 is an upstream regulator of $M Y C^{17}$. In leukaemic BM cells, forced expression of miR-22, but not miR-22 mutant, significantly repressed expression of MYC downstream oncogenic targets Bmi1, Fasn and Hmgal (refs 36-38); the repression could be reversed by coexpressing MYCBP or FLT3 (Supplementary Fig. 3i,j). Those three genes all showed significant inverse correlations with miR22 in expression in human AML (Supplementary Table 2; Supplementary Fig. 3f). The miR-22-induced repression of Bmil, Cdk6 and Hmgal at the protein level was also observed (Supplementary Fig. 3c). 


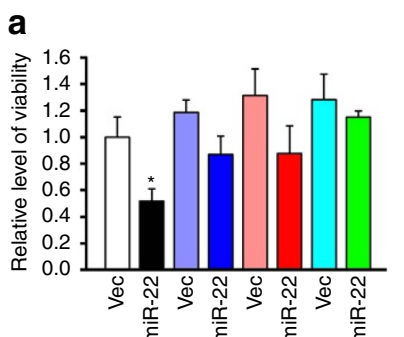

$\overline{\mathrm{Ctrl}} \overline{\mathrm{CRTC}} 1 \overline{\mathrm{FLT}} \mathrm{MYCBP}$

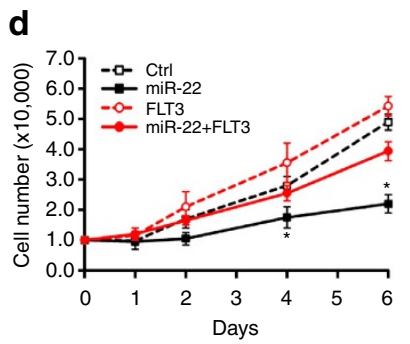

g
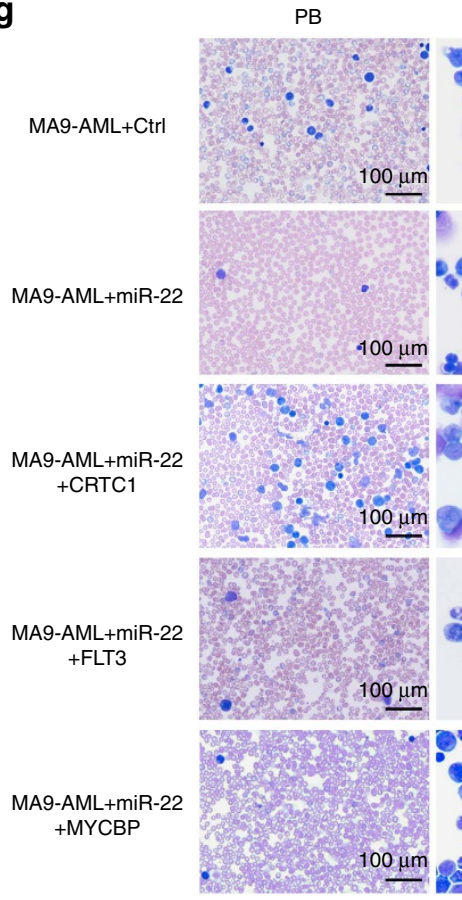

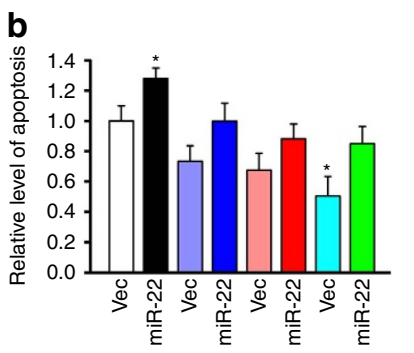

C

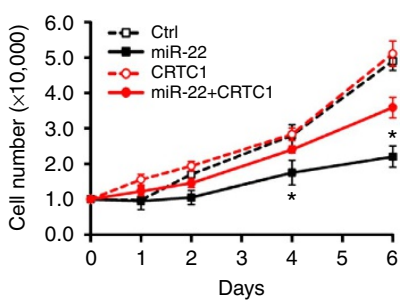

$\overline{\mathrm{Ctrl}} \overline{\mathrm{CRTC}} 1 \overline{\mathrm{FLT3}} \mathrm{MYCBP}$

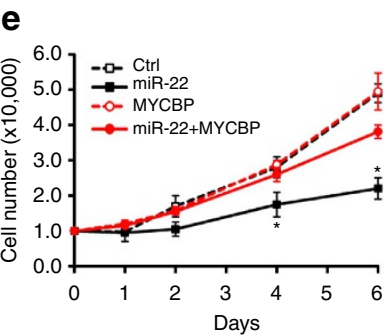

$\mathbf{f}$

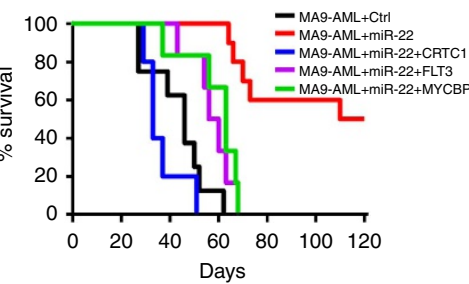

BM

SP
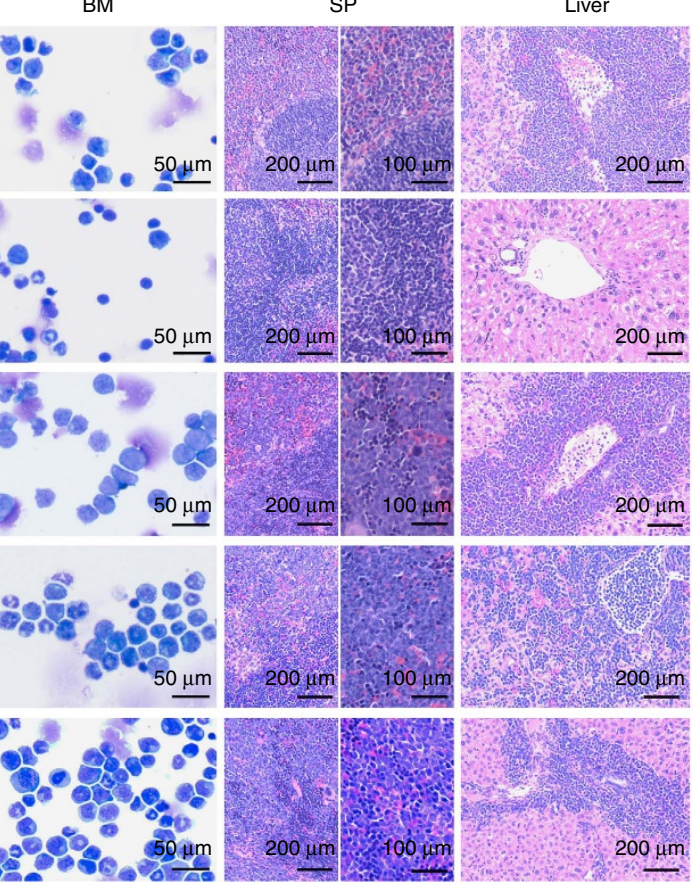

Figure 4 | Multiple onocgenes are functionally important targets of miR-22 in AML. (a,b) Relative viability (a) and apoptosis (b) levels of MONOMAC-6 cells transfected with MSCV-PIG-CRTC1, -FLT3 or -MYCBP alone, or together with MSCVneo-miR-22. Values were detected $48 \mathrm{~h}$ post transfection. (c-e) Rescue effects of CRTC1 (c), FLT3 (d) and MYCBP (e) on the inhibition of MONOMAC-6 growth mediated by miR-22. Cell counts at the indicated time points are shown. Mean \pm s.d. values are shown. ${ }^{\star} P<0.05$, $t$-test. (f) In vivo rescue effects of $C R T C 1, F L T 3$ and MYCBP on the inhibition of $M L L-A F 9$-induced leukemogenesis mediated by miR-22. The secondary recipients were transplanted with BM blast cells of the primary MLL-AF9 leukaemic mice retrovirally

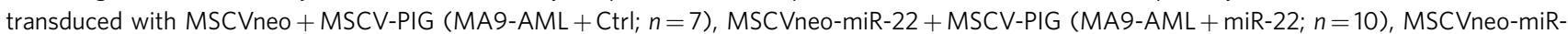
22 + MSCV-PIG-CRTC1 (MA9-AML + miR-22 + CRTC1; $n=5$ ), MSCVneo-miR-22 + MSCV-PIG-FLT3 (MA9-AML + miR-22 + FLT3; $n=6$ ) or MSCVneomiR-22 + MSCV-PIG-MYCBP (MA9-AML + miR-22 + MYCBP; $n=6$ ). Kaplan-Meier curves for all the five groups of transplanted mice are shown. MA9-AML + Ctrl versus MA9-AML + miR-22, P<0.001 (log-rank test); MA9-AML + Ctrl versus any other groups, $P>0.05$ (log-rank test).

(g) Wright-Giemsa stained $\mathrm{PB}$ and $\mathrm{BM}$, and $\mathrm{H} \& \mathrm{E}$ stained spleen and liver of the secondary leukaemic mice.

DNA copy-number loss of miR-22 gene locus in AML. DNA copy-number loss of tumour-suppressor gene(s) is a hallmark of many cancers including AML ${ }^{39}$. Deletions of human chromosome 17 band p13.3, where miR-22 is located, have been frequently reported in various types of leukaemia, lymphoma and solid tumours ${ }^{40-43}$. Here we found that $18 \%(9 / 50)$ of the AML samples showed deletions (mostly hemizygous) of the miR-22 gene locus (Supplementary Fig. 4a,b). Similarly, in analysis of three publically available AML data sets, we found that $7-9 \%$ of the AML cases carried loss of one or even two alleles of the miR-22 locus (Supplementary Fig. 4c). Therefore, DNA copy-number loss in miR-22 gene locus does exist in AML cases.

Expression of miR-22 is epigenetically repressed in AML. It was reported that TET2 is repressed by miR-22 as its direct target in 

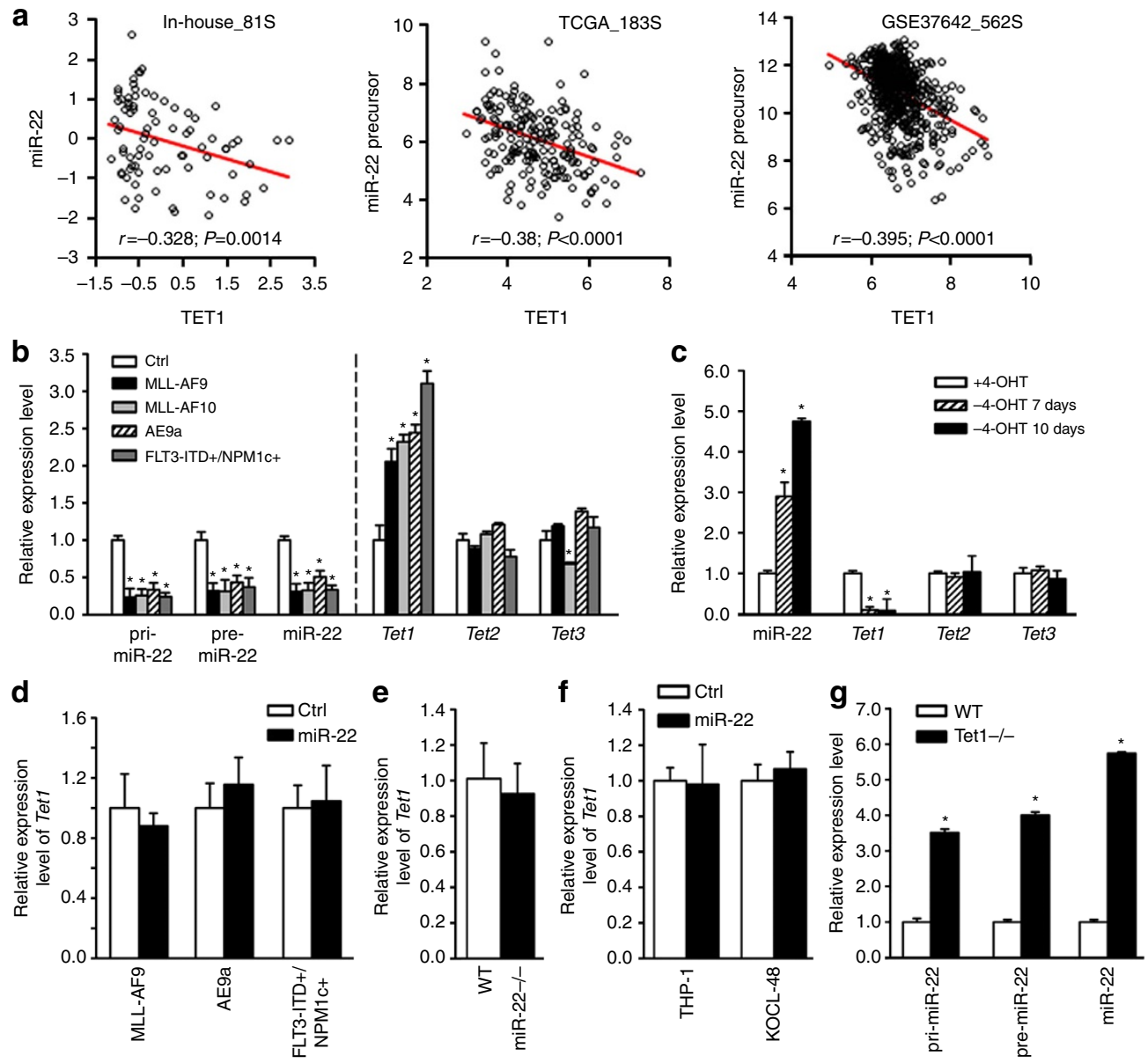

Figure 5 | Transcriptional correlation between miR-22 and TET1. (a) Correlation between the expression levels of miR-22 and TET1 in three independent AML patient databases. All expression data were $\log (2)$ transformed; the data in In-house_81S were also mean-centred. The correlation coefficient ( $r$ ) and $P$ values were detected by 'Pearson Correlation', and the correlation regression lines were drawn with the 'linear regression' algorithm. (b) Expression of pri-, pre- and mature miR-22, and Tet1/2/3 in colony-forming cells of wild-type mouse BM progenitors retrovirally transduced with MSCVneo (Ctrl), MSCVneo-MLL-AF9 (MLL-AF9), MSCVneo-MLL-AF10 (MLL-AF10) or MSCVneo-AE9a (AE9a), or of FLT3-ITD/NPM1C ${ }^{+}$mouse BM progenitors transduced with MSCVneo (FLT3-ITD +/NPM1c + ). (c) Expression of miR-22 and Tet1/2/3 in MLL-ENL-ERtm cells. Expression levels were detected at the indicated time points post 4-OHT withdrawal. (d) Effect of miR-22 overexpression on Tet1 expression in colony-forming cells with MLL-AF9, AE9a or FLT3-ITD/ $\mathrm{NPM1C}^{+}$. (e) Expression of Tet1 in BM progenitor cells of 6 -weeks old miR-22-/- or wild-type mice. (f) Effect of miR-22 overexpression on TET1 expression in THP-1 and KOCL-48 AML cells $48 \mathrm{~h}$ post transfection. (g) Expression of pri-, pre- and mature miR-22 in BM progenitor cells of 6-weeks old $\mathrm{Tet}^{-1}{ }^{-}$or wild-type mice. Mean \pm s.d. values are shown. ${ }^{\star} P<0.05, t$-test.

breast cancer and $\mathrm{MDS}^{15,16}$. Here we analysed the expression patterns of TET1/2/3 and miR-22 in three independent AML patient data sets ${ }^{21,22,44}$ (Supplementary Table 3). To our surprise, we found that TET2 (and likely also TET3) exhibited a positive correlation, whereas only TET1 exhibited a negative correlation, with miR-22 in expression in AML (Supplementary Table 3; Fig. 5a). The primary, precursor and mature miR-22 levels were all significantly downregulated by $M L L-A F 9, M L L-A F 10, A E 9 a$ and FLT3-ITD/NPM1c ${ }^{+}$in colony-forming cells, while Tet 1 (but not Tet2 or Tet3) was upregulated (Fig. 5b). Conversely, in MLL-ENL-ERtm cells ${ }^{31}$, Tet 1, but not Tet2 or Tet3, was downregulated when miR-22 was upregulated after withdrawal of 4-OHT (Fig. 5c). Thus, Tet1, instead of Tet2, exhibited an inverse correlation with miR-22 in expression in both human and mouse leukaemic cells. Tet1 also exhibits an inverse correlation with miR-22 in expression during mouse normal myeloid differentiation (Supplementary Fig. 5a). Furthermore, as miR-22 and TET1 were expressed at a significantly higher and lower level, respectively, in human normal $\mathrm{CD} 33^{+}$myeloid progenitor cells than in CD34 ${ }^{+}$HSPCs or MNCs (see Fig. 1a and ref. 14), the inverse expressional correlation between miR-22 and TET1 likely also existed in human normal hematopoietic cells.

However, forced expression of miR-22 caused no noticeable changes in Tet1 expression in MLL-AF9, AE9a or FLT3-ITD/ NPM1c ${ }^{+}$colony-forming cells (Fig. 5d). Similarly, neither miR-22 knockout nor overexpression resulted in any significant changes of Tet1/TET1 expression (Fig. 5e,f). In contrast, Tet1 knockout remarkably increased the levels of pri-, pre- and mature miR-22 (Fig. 5g). Thus, our data suggest that miR-22 is a downstream target of and negatively regulated by Tet1, and that there is no negative feedback of miR-22 on Tet1 expression.

Tet1 has been shown to cooperate with Polycomb repressive complex 2 (PRC2) components and cofactors, such as Ezh2 and Sin3a, to repress transcription of their co-target genes in mouse embryonic stem cells ${ }^{8,9}$. Our luciferase reporter assay showed that forced expression of Tetl significantly repressed the transcriptional activity controlled by the miR-22 promoter ${ }^{45}$, suggesting that miR-22 is a direct repressed target of Tet1 (Fig. 6a). In an all-trans retinoic acid (ATRA)-induced THP-1/t $(9 ; 11)$ monocytic differentiation model ${ }^{46}$, we showed 
a

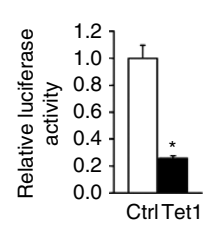

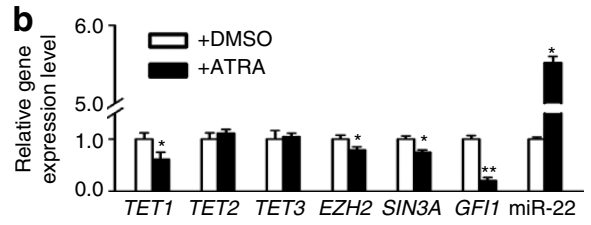

C

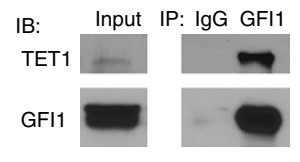

d

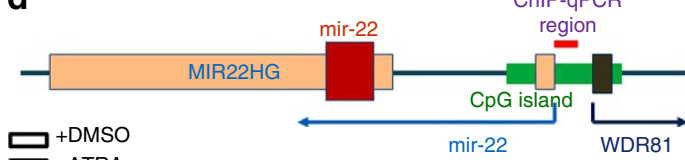

+ATRA
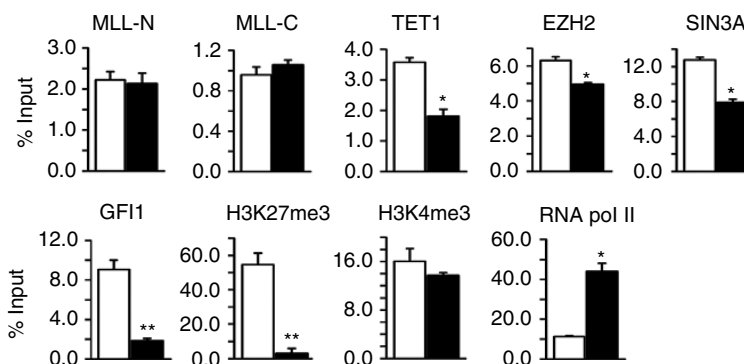

SIN3A
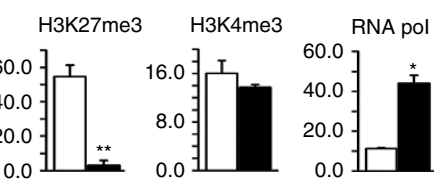

e

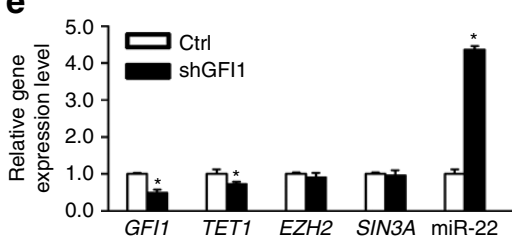

f

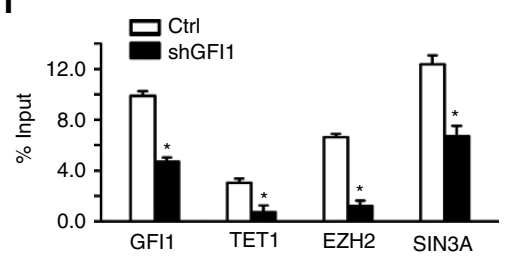

g

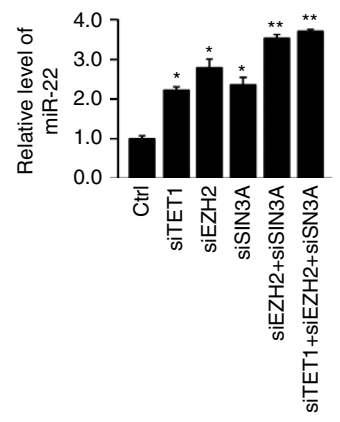

h

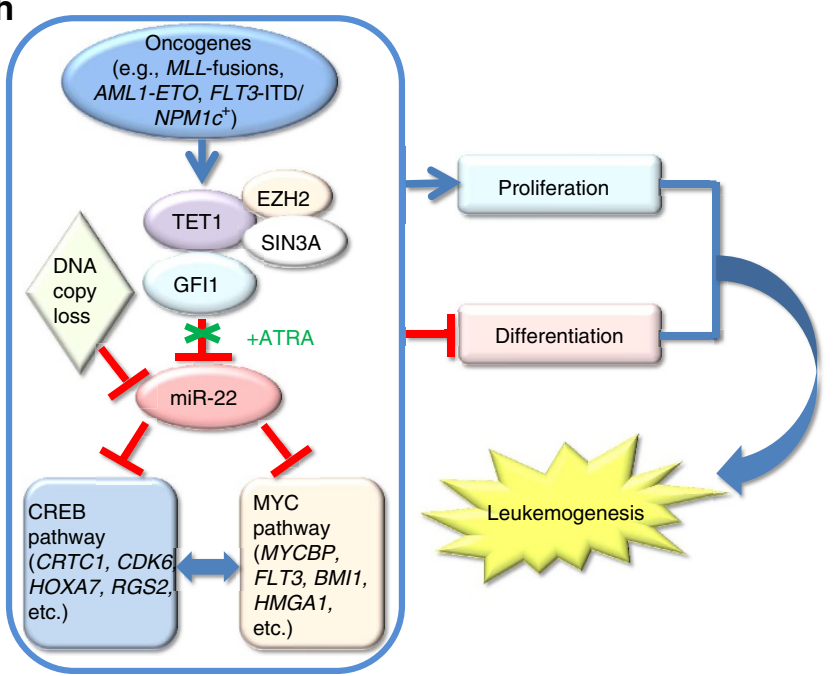

Figure 6 | TET1 mediates epigenetic repression of miR-22 transcription. (a) Tet1 targets miR-22 promoter region $(-1,100 /+55 \mathrm{bp})$, as detected by luciferase reporter assay $48 \mathrm{~h}$ post transfection in HEK293T cells. (b) Expression of TET1/2/3, EZH2, SIN3A, GFI1 and miR-22 in THP-1 cells $72 \mathrm{~h}$ post treatment with $1 \mu \mathrm{M}$ ATRA or DMSO control. (c) Co-immunoprecipitation assay showing the binding of endogenous GFI1 and TET1 in THP1 cells. (d) ChIPqPCR analyses of the promoter region of miR-22 in THP- 1 cells $72 \mathrm{~h}$ post treatment with $1 \mu \mathrm{M}$ ATRA or DMSO. Upper panel: PCR site on the CpG-enriched region of miR-22 gene locus. Note: miR-22 is coded within the second exon of a long non-coding RNA (MIR22HG), which represents the primary transcript of miR-22. Lower panels: enrichment of MLL-N terminal (for both wild-type MLL and MLL-fusion proteins), MLL-C terminal (for wild-type MLL), TET1, EZH2, SIN3A, GFI1, H3K27me3, H3K4me3 or RNA pol Il at miR-22 promoter region. (e) Expression levels of TET1, EZH2, SIN3A and miR-22 in GFI1 knockdown cells. (f) ChIP-qPCR analyses of the promoter region of miR-22 in THP-1 cells transduced with GFI1 shRNA or control shRNA. Enrichment of GFI1, TET1, EZH2 and SIN3A are shown. (g) Effects of knockdown of TET1, EZH2 and/or SIN3A on miR-22 expression. The expression level of miR-22 was detected in THP-1 cells $72 \mathrm{~h}$ post transfection with siRNAs targeting TET1, EZH2 and/or SIN3A. Mean \pm s.d. values are shown. ${ }^{\star} P<0.05 ;{ }^{* \star} P<0.01$ ( $t$-test). (h) Schematic model of the regulatory pathway involving miR-22 in AML and ATRA treatment.

that on treatment with ATRA, TET1 (but not TET2 or TET3), EZH2 and SIN3A were significantly downregulated, accompanied by the upregulation of miR-22 (Fig. 6b). WDR81 is the gene that is located closely (within $500 \mathrm{bp}$ ) but oppositely to the miR-22 gene loci (Fig. 6d). We also tested the potential influence of ATRA on the expression level of WDR81 in the same model. ATRA treatment showed no significant effects on WDR81 level (Supplementary Fig. 5b), suggesting that TET1 specifically inhibits the transcription of miR-22, but not its neighbouring gene with the opposite orientation.
While miR-22 expression level had a more than fivefold increase on ATRA treatment, the degrees of decrease in expression levels of TET1, EZH2 and SIN3A are relatively mild (though statistically significant) (Fig. 6b). To identify additional transcription factor(s) that is (are) more responsive to ATRA treatment and can facilitate TET1 binding to miR-22 promoter region, we searched for transcription factors that have evolutionarily conserved binding sites within the $\mathrm{CpG}$ island of miR-22 locus. Among a set of such transcription factors (including GFI1, STAT, PAX4, HMX1 and SRF), only GFI1 exhibited a significant 

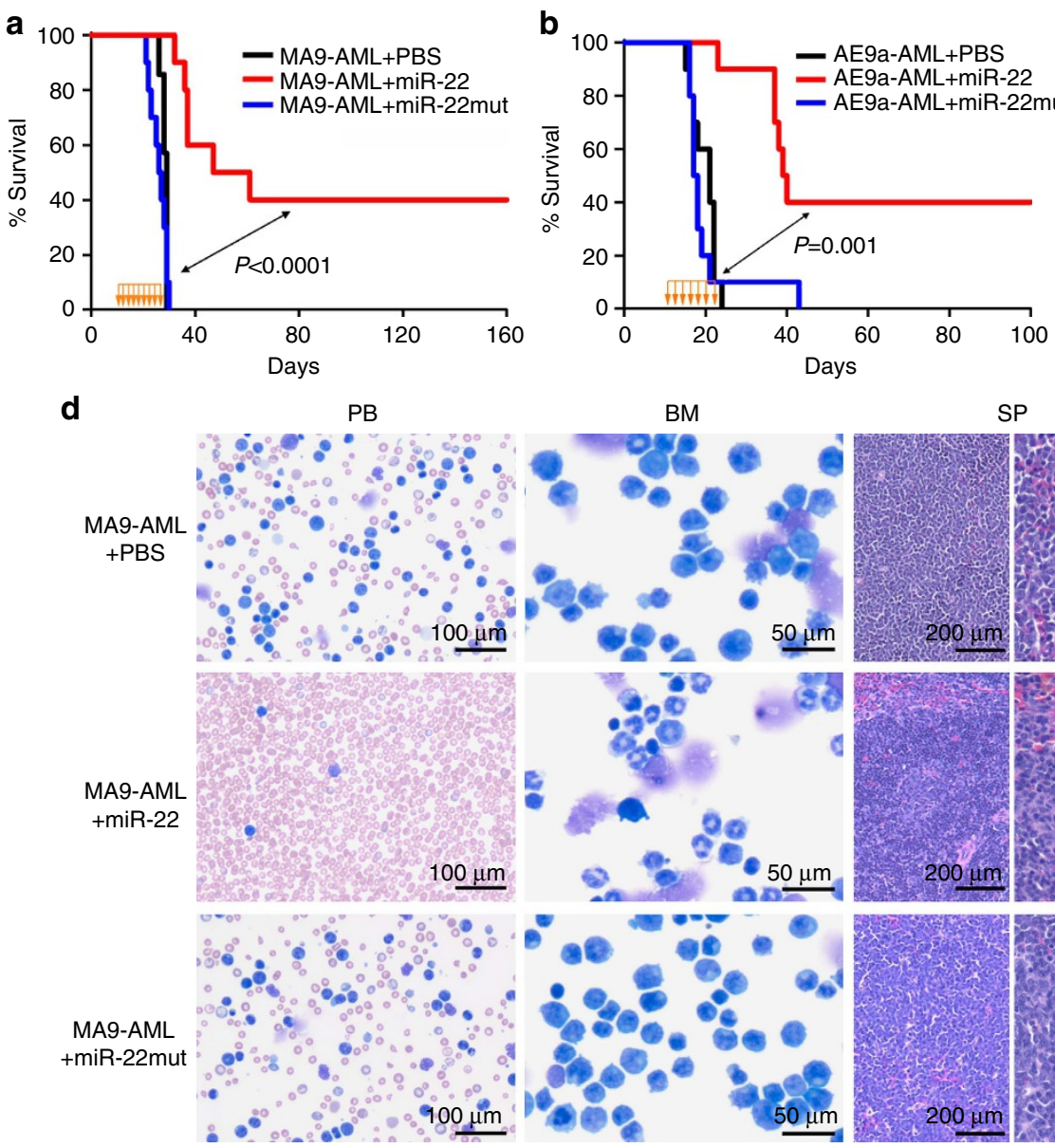

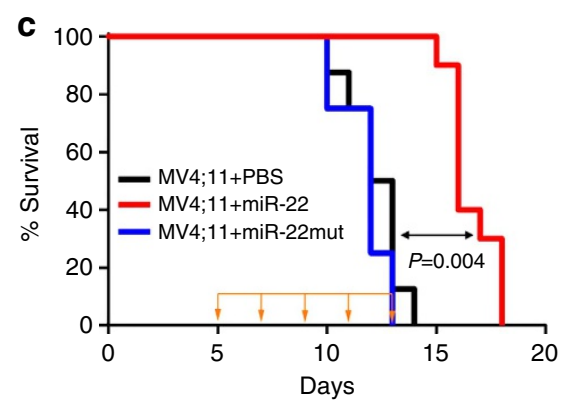

SP
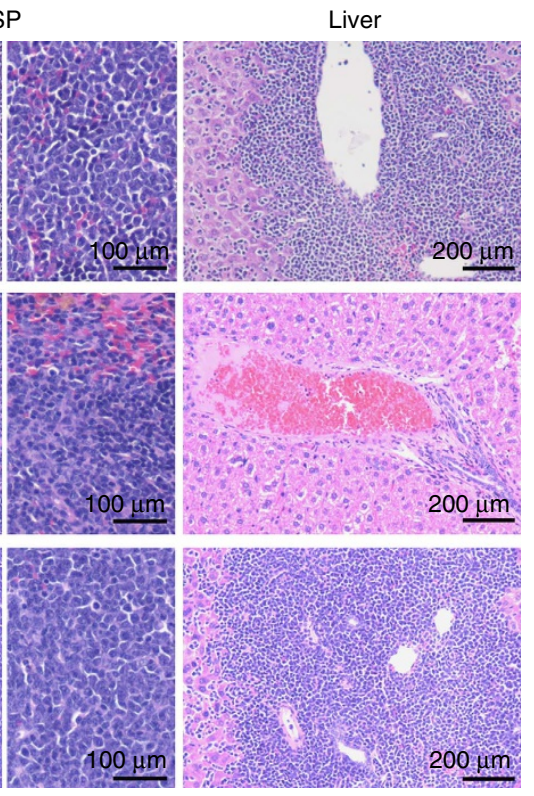

Figure 7 | Therapeutic effect of miR-22-nanoparticles in treating AML. (a,b) Primary leukaemia BM cells bearing MLL-AF9 (a) or AE9a (b) were transplanted into sublethally irradiated secondary recipient mice. After the onset of secondary AML (usually 10 days post transplantation), the recipient mice were treated with PBS control, or $0.5 \mathrm{mg} \mathrm{kg}^{-1}$ miR-22 or miR-22 mutant RNA oligos formulated with G7 PAMAM dendrimer nanoparticles, i.v., every other day, until the PBS-treated control group all died of leukaemia. (c) NSGS mice ${ }^{49}$ were transplanted with MV4;11/t(4;11) AML cells. Five days post transplantation, these mice started to be treated with PBS control, miR-22 or miR-22 mutant nanoparticles at the same dose as described above.

Kaplan-Meier curves are shown; the drug administration period and frequency were indicated with yellow arrows. The $P$ values were detected by log-rank test. (d) Wright-Giemsa stained PB and BM, and H\&E stained spleen and liver of the MLL-AF9-secondary leukaemic mice treated with PBS control, miR-22 or miR-22 mutant nanoparticles.

inverse correlation with miR-22 in expression in all large-scale AML cohorts (Supplementary Table 3). Interestingly, it was reported previously that ATRA treatment could significantly diminish the binding of GFI1 to the loci of many of its target genes, for example, IL-6R, JAK3, E2F6 and so on ${ }^{47}$. Thus, we chose GFI1 for further studies.

Notably, we found that ATRA treatment substantially reduced the transcription level of GFI1 in AML cells and its decrease degree was greater than that of TET1, EZH2 or SIN3A (Fig. 6b). We further showed that GFI1 is a binding partner of TET1 in both THP1 and HEK-293T cells (Fig. 6c; Supplementary Fig. 5c). ATRA treatment remarkably reduced the binding of GFI1, TET1, EZH2 and SIN3A, but not that of MLL protein, to the miR-22 promoter region (Fig. 6d). H3K27me3 modifications and RNA polymerase II (RNA pol II) occupancy were significantly decreased and increased, respectively, while $\mathrm{H} 3 \mathrm{~K} 4 \mathrm{Me} 3$ modifications showed no significant change (Fig. 6d). Noticeably, the enrichment of GFI1 to this region was diminished by ATRA to a greater degree than that of TET1, EZH2 or SIN3A (Fig. 6d), suggesting that GFI1 might be the primary effector of ATRA treatment in regulating miR-22 expression. Consistently, knockdown of GFI1 resulted in a dramatic increase in miR-22 expression ( $>4$ fold; Fig. 6e), associated with a significant decrease in the binding of TET1, EZH2, SIN3A and GFI1 itself to the miR-22 promoter region (Fig. $6 \mathrm{f}$ ). Knockdown of expression of TET1, EZH2 or SIN3A resulted in a 2-3-fold increase in miR-22 expression (Fig. 6g), with no effects on GFI1 expression (Supplementary Fig. 5d); only their combinational knockdown could cause a similar level of increase in miR-22 expression (Fig. 6g; Supplementary Fig. 5e) to that induced by GFI1 knockdown (Fig. 6e). As expected, the expression level of WDR81 was not changed on knockdown of GFI1, TET1, EZH2 or SIN3A (Supplementary Fig. 5f,g). The above data suggest that GFI1, TET1, EZH2 and SIN3A are all involved in transcriptional repression of miR-22 expression; and when treated with ATRA, GFI1 likely functions as the primary effector that facilitates the binding of TET1/EZH2/SIN3A complex to the miR-22 promoter region.

As TET1 is a methylcytosine dioxygenase ${ }^{8-10}$, we conducted bisulfite sequencing analysis to investigate whether TET1 affects the methylation status of the miR-22 promoter. The analysis showed that the miR-22 promoter was hypomethylated in AML cells, no matter with ATRA treatment or not (Supplementary Fig. 5h). The hypomethylated status of the miR-22 promoter region in various AML was confirmed by analysing the TCGA_194S data set with DNA methylation information 
(Supplementary Fig. 5i). The methylation status of the miR-22 promoter showed no significant correlation with miR-22 expression level in AML (Supplementary Fig. 5j). These data suggest that the hypomethylation status of miR-22 promotor region does not lead to a high-level expression of miR-22 in AML, and TET1-mediated repression of miR-22 transcription is unlikely related to its methylcytosine dioxygenase activity.

The miR-22-associated regulatory circuit in AML. The above data suggest that repression of miR-22 in AML is attributed to both DNA copy-number loss and especially TET1-mediated transcriptional suppression. Interestingly, among the nine AML samples with DNA copy-number loss of miR-22 locus (Supplementary Fig. 4a,b), the AML samples with both copynumber loss and TET1 overexpression generally exhibited a more significant repression of miR-22 expression than those with copynumber loss alone (Supplementary Fig. 5k). Thus, those two mechanisms are not mutually exclusive and can have synergistic effect on reducing miR-22 expression.

Collectively, our studies revealed a previously unappreciated genetic/epigenetic regulatory circuit in AML (Fig. 6h). In this circuit, oncogenic fusion genes or gene mutants (for example, $M L L$-fusions, $A E 9 a$ and FLT3-ITD/NPM1c ${ }^{+}$) function as the 'drivers'. They promote the expression of TET1, which in turn, through recruiting polycomb cofactors such as EZH2 and SIN3A, represses the transcription of miR-22 by increasing $\mathrm{H} 3 \mathrm{~K} 27 \mathrm{me} 3$ and decreasing RNA Pol II binding at the miR-22 promoter. When AML cells are treated with ATRA, ATRA substantially diminishes the enrichment of GFI1, a binding partner of TET1, at the miR-22 promoter, and thereby inhibits the recruitment of the TET1/EZH2/SIN3A complex to this region. In addition, miR-22 can also be compromised in its function by genetic mechanism(s) such as DNA copy-number loss in a portion (7-18\%) of the AML cases. The inactivation of miR-22 results in the de-repression of its critical oncogenic targets such as CRCT1, MYCBP and FLT3, and thereby the activation of both CREB and MYC signalling pathways, leading to cell transformation and leukemogenesis.

Restoration of miR-22 expression and function to treat AML. To investigate the therapeutic potential of restoration of miR-22 expression/function in treating AML, we employed amine-terminated, generation 7 (G7) poly(amidoamine) (PAMAM) dendrimers (Supplementary Fig. 6a,b), an effective non-viral gene delivery vector with minimal side effects ${ }^{48}$. Nanoparticles carrying miR-22 oligos significantly delayed AML progression in both $M L L-A F 9$ and $A E 9 a$-induced secondary leukaemic recipients (Fig. 7a,b). Notably, at least $40 \%$ of the treated mice seemed to be completely cured by the miR-22 nanoparticles as the pathological morphologies in $\mathrm{PB}, \mathrm{BM}$, spleen and liver tissues all became normal (Fig. 7d; Supplementary Fig. 6c). In contrast, the miR-22 mutant nanoparticles exhibited no significant therapeutic effect (Fig. 7a,b,d; Supplementary Fig. 6c). As expected, miR-22 oligos, but not miR-22 mutant oligos, significantly inhibited expression of its critical targets (that is, Crct1, Flt3 and Mycbp) in BM cells of the treated mice (Supplementary Fig. 6d). The miR22-nanoparticles showed no noticeable effects on blood cell lineages (Supplementary Table 4).

We then tested the miR-22 nanoparticles in a xenotransplantation model ${ }^{49}$. Similarly, the nanoparticles carrying miR-22 oligos, but not miR-22 mutant, significantly delayed AML progression induced by human MV4;11/t $(4 ; 11)$ cells (Fig. 7c). The miR-22-nanoparticle administration also resulted in less aggressive leukaemic pathological phenotypes in the recipient mice (Supplementary Fig. 6e). Thus, our studies demonstrated the therapeutic potential of using miR-22-based nanoparticles to treat AML.

\section{Discussion}

It remains poorly understood how TET proteins mediate gene regulation in cancer. Here we show that in de novo AML, it is TET1, but not TET2 (a reported direct target of miR-22 in MDS and breast cancer ${ }^{15,16}$ ), that inversely correlates with miR-22 in expression and negatively regulates $\mathrm{miR}-22$ at the transcriptional level. Likely together with GFI1, TET1 recruits polycomb cofactors (for example, EZH2/SIN3A) to the miR-22 promoter, leading to a significant increase in H3K27me3 occupancy and decrease in RNA pol II occupancy at that region, and thereby resulting in miR-22 repression in AML cells; such a repression can be abrogated by ATRA treatment. Thus, our study uncovers a novel epigenetic regulation mechanism in leukaemia involving the cooperation between TET1/GFI1 and polycomb factors.

Besides GFI1, it was reported that LSD1 is also a binding partner of TET1 (ref. 50). Interestingly, LSD1 is known as a common binding partner shared by TET1 and GFI1, and mediates the effect of GFI1 on hematopoietic differentiation ${ }^{51,52}$. Thus, it is possible that LSD1 might also participate in the transcriptional repression of miR-22 as a component of the GFI1/TET1 repression complex.

We previously reported that TET1 cooperates with MLL fusions in positively regulating their oncogenic co-targets in $M L L$-rearranged $\mathrm{AML}^{14}$. Here we show that TET1 can also function as a transcriptional repressor (of a miRNA) in cancer. The requirement of TET1-mediated regulation on expression of its positive (for example, HOXA/MEIS1/PBX3) ${ }^{14}$ or negative (for example, miR-22) downstream effectors in leukemogenesis likely explains the rareness of TET1 mutations in $\mathrm{AML}^{53}$, and highlights its potent oncogenic role in leukaemia.

The aberrant activation of both CREB and MYC signalling pathways has been shown in $\mathrm{AML}^{24-26,54,55}$, but the underlying molecular mechanisms remain elusive. Our data suggest that the activation of these two signalling pathways in AML can be attributed, at least in part, to the repression of miR-22, which in turn, results in the de-repression of CRTC1 (CREB pathway), FLT3 and MYCBP (MYC pathway), and leads to the upregulation of oncogenic downstream targets (for example, CDK6, HOXA7, $B M I 1, F A S N$ and HMGA1) and downregulation of tumoursuppressor downstream targets (for example, RGS2).

In summary, we uncover a TET1/GFI1/EZH2/SIN3A $\dashv$ miR$22-\mathrm{CREB}-\mathrm{MYC}$ signalling circuit in de novo AML, in which miR-22 functions as a pivotal anti-tumour gate-keeper, distinct from its oncogenic role reported in MDS or MDSderived AML ${ }^{16}$. Thus, our study together with the study of Song et al. ${ }^{16}$ highlight the complexity and functional importance of miR-22-associated gene regulation and signalling pathways in hematopoietic malignancies, and may provide novel insights into the genetic/epigenetic differences between de novo AML and MDS.

Our findings also highlight the possibility of using miR-22based therapy to treat AML patients. Our proof-of-concept studies demonstrate that the nanoparticles carrying miR-22 oligos significantly inhibit AML progression and prolong survival of leukaemic mice in both BMT and xeno-transplantation models. Notably, miRNA-based nanoparticles have already entered clinical trials ${ }^{56}$. It would be important, in the future, to further test the combination of miR-22-carrying nanoparticles (or smallmolecule compounds that can induce endogenous expression of miR-22) with standard chemotherapy agents (cytosine arabinoside and anthracycline), or with the emerging small molecule inhibitors against MYC and/or CREB pathway effectors, to achieve optimal anti-leukaemia effect with minimal side effects. Overall, our results suggest that restoration of miR-22 expression/ function (for example, using miR-22-carrying nanoparticles or small-molecule compounds) holds great therapeutic potential to treat AML, especially those resistant to current therapies. 


\section{Methods}

AML and MDS samples and cell lines. The AML and MDS patient samples were obtained at the time of diagnosis with informed consent at the University of Chicago Hospital (UCH), and were approved by the University of Chicago Hospital Institutional Review Board (UCHIRB). All patients were treated according to the protocols of the corresponding institutes/hospitals. THP-1, KOCL48, MV4;11, MEF and HEK-293T cells were purchased from ATCC (Manassas, VA) and maintained in the lab. The MLL-ENL-ER cell line was a gift from Dr Robert Slany ${ }^{31}$. All the cell lines were tested for mycoplasma contamination yearly using a PCR Mycoplasma Test Kit (PromoKine).

Preparation of AML and MDS samples. The primary AML and MDS samples were stored in liquid nitrogen until used. Blasts and mononuclear cells were purified by use of NycoPrep 1.077A (Axis-Shield, Oslo, Norway) according to the manufacturer's manual.

Human normal hematopoietic control cell samples. The MNC normal control samples were isolated from normal BM cells purchased from AllCells, LLC (Emeryville, CA) by use of NycoPrep 1.077A (Axis-Shield, Oslo, Norway) according to the manufacturer's manual.

Mouse normal BM cell population sorting. As described previously ${ }^{23}$, wild-type C57BL6/J mice were used for the sorting. All laboratory mice were maintained in the animal facility at the University of Chicago and the University of Cincinnati. All experiments on mice in our research protocol were approved by Institutional Animal Care and Use Committee (IACUC) of the University of Chicago and the University of Cincinnati.

The long-term hematopoietic stem cells (LT-HSCs; $\mathrm{Lin}^{-} \mathrm{Sca}^{+}{ }^{+} \mathrm{c}-\mathrm{Kit}^{+}{ }^{+} \mathrm{Flk}_{2}$, $\mathrm{LSKF}^{-}$), short-term HSCs (ST-HSCs; $\mathrm{Lin}^{-} \mathrm{Scal}{ }^{+}{ }^{\mathrm{c}-\mathrm{Kit}^{+}}{ }^{+} \mathrm{Flk}_{2}{ }^{+}, \mathrm{LSKF}^{+}$), and the committed progenitors $\left(\mathrm{CP}, \mathrm{Lin}^{-} \mathrm{Scal}{ }^{-} \mathrm{c}^{-} \mathrm{Kit}^{+}\right)$were enriched by lineage ${ }^{+}$cell depletion (EasySep Mouse Hematopoietic Progenitor Cell Enrichment Kit; StemCell Technologies, Vancouver, BC), and purified by FACSAria flow cytometer (BD Biosciences, San Jose, CA) sorting after $20 \mu \mathrm{l}$ per test fluorescein isothiocyanate-conjugated lineage (FITC-Lin) cocktail (including FITC-CD3 (17A2), FITC-B220 (RA3-6B2), FITC-CD11b (M1/70), FITC-TER-119 (TER-119), FITC-Gr1 (RB6-8C5)), $5 \mu \mathrm{g} \mathrm{ml}^{-1}$ phycoerythrin (PE)-Scal (D7), $1.5 \mu \mathrm{g} \mathrm{ml}^{-1}$ APC-c-Kit (ACK2) and $20 \mu \mathrm{g} \mathrm{ml}^{-1}$ PE-Cy5.5-Flk2 (A2F10) staining. Then, $\mathrm{Grl}^{+} \mathrm{Macl}^{+}$myeloid cells and B220 ${ }^{+} \mathrm{B}$ cells were sorted from BM cells after $2.5 \mu \mathrm{g} \mathrm{ml}^{-1}$ FITC-Gr1 (RB6-8C5), $2.5 \mu \mathrm{g} \mathrm{ml}^{-1} \mathrm{PE}-\mathrm{Macl}$ (M1/70) and $5 \mu \mathrm{g} \mathrm{ml}^{-1}$ APC-B220 (RA3-6B2) staining. All fluorescent antibodies used were purchased from eBioscience (San Diego, CA).

RNA extraction and quantitative RT-PCR. Total RNA was extracted with the miRNeasy extraction kit (Qiagen, Valencia, CA) and was used as template to synthesize complementary DNA for quantitative reverse transcription PCR (qRT-PCR) analysis in a 7900HT real-time PCR system (Applied Biosystems, Foster City, CA). TaqMan qPCR assay was performed to validate the differential expression patterns of miR-22 using commercial kits from Applied Biosystems (Cat. no. 4427975). Sequences for the controls are: sno202: $5^{\prime}$-GCTGTACTGACT TGATGAAAGTACTTTTGAACCCTTTTCCATCTGATG-3 ${ }^{\prime}$; RNU6B: $5^{\prime}$-CGCA AGGATGACACGCAAATTCGTGAAGCGTTCCATATTTTT-3'. qPCR with SYBR Green dye (Qiagen) was used to determine expression of mRNA genes. snoRNA202, RNU48, Gapdh or GAPDH were used as endogenous controls for qPCR of miRNA and mRNA, respectively. Each sample was run in triplicate. qPCR primers are available on request. For determining the miR-22 DNA locus copy number, TaqMan $\mathrm{qPCR}$ assay was used as described previously ${ }^{57}$.

microRNA microarray and exon array assays. As described previously ${ }^{17,23}$, our miRNA expression profiling assay of 85 (including $10 \mathrm{t}(8 ; 21), 9 \operatorname{inv}(16), 9 \mathrm{t}(15 ; 17)$, $10 M L L$-rearranged, $11(+8), 29$ normal karyotype and 7 others $)$ AML samples, and 15 human normal BM samples was performed by Exiqon (Woburn, MA) using the miRCURY LNA arrays (v10.0; covering 757 human miRNAs). The 15 normal $\mathrm{BM}$ controls included six CD34 + hemtopoietic stem/progenitor, five CD33 + myeloid progenitor and four MNC samples. In terms of patient samples, MNCs isolated from the BM or PB cells of the $85 \mathrm{AML}$ patients were used. The expression values are $\log 2(\mathrm{Hy} 3 / \mathrm{Hy} 5)$ ratios, which were obtained on the basis of the normalized data where replicated measurements on the same slide have been averaged. In addition, as described previously ${ }^{14,17,23}$, a total of 100 human AML (including $30 \mathrm{t}(8 ; 21), 27 \mathrm{inv}(16), 31 \mathrm{t}(15 ; 17)$ and $12 \mathrm{MLL}$-rearranged), and 9 normal BM samples (including three each of CD34 ${ }^{+}$hematopoietic stem/ progenitor, $\mathrm{CD}_{3}{ }^{+}$myeloid and $\mathrm{MNC}$ samples) were analysed by use of Affymetrix GeneChip Human Exon 1.0 ST arrays (Affymetirx, Santa Clara, CA). The QC test and Affymetrix exon array assays were done in the core facility of National Human Genome Research Institute, NIH (Bethesda, MD). Robust multiarray average (RMA) ${ }^{58}$ was used for the data normalization with Partek Genomics Suite (Partek Inc., St Louis, MI). The complete microarray data set has been deposited in the GEO database under the accession codes GSE34184 and GSE30285.
Among the above 100 human AML samples, 81 samples (that is, the In-house 81 ; including $29 \mathrm{t}(8 ; 21), 26 \operatorname{inv}(16)$ and $26 \mathrm{t}(15 ; 17)$ AML) have been also included in the Exiqon microRNA array assay ${ }^{21}$. The microarray data set of those 81 AML samples has been deposited in GEO database under the accession code GSE27370.

Affymetrix gene arrays of mouse samples. As described previously ${ }^{17}$, a total of 15 mouse BM samples including 6 primary (including three each of negative control and $M L L-A F 9$ ) and 9 secondary (including three negative control and six $M L L-A F 9)$ obtained from the in vivo mouse BM reconstitution assays were analysed by use of Affymetrix GeneChip Mouse Gene 1.0 ST Array (Affymetirx). The RNA quality control, cDNA amplification, hybridization and image scan were conducted in the Functional Genomics Facility of the University of Chicago. RMA $^{58}$ was used for the data normalization with Partek Genomics Suite (Partek Inc.). The microarray data set of those 15 mouse AML samples has been deposited at GEO database (GSE34185)

Affymetrix microarray assay of GSE37642_562S set. The GSE37642_562S set $(n=562)$ AML samples (including $30 \mathrm{t}(8 ; 21), 38 \mathrm{inv}(16), 24 \mathrm{t}(15 ; 17), 38$ $M L L$-rearranged, $6 \operatorname{del}(5 q), 16 \operatorname{del}(7 q), 15 \operatorname{inv} 3 / t(3 / 3), 74$ complex, 199 normal karyotype and 122 others) were analysed by use of Affymetrix Human Genome U133Plus2.0 GeneChips $(n=140)$ or Affymetrix Human Genome U133A and B $(\mathrm{U} 133 \mathrm{~A}+\mathrm{B} ; n=422)$ GeneChips. RMA method ${ }^{58}$ was used for data normalization. The AML samples were collected by the German AMLCG study group. Part of the microarray data have been reported previously ${ }^{44}$. The GEO ID of the entire data set is GSE37642.

TCGA data sets. The Cancer Genome Atlas (TCGA) AML database ${ }^{22}$ includes mRNA gene expression profiling data of 183 adult de novo AML cases (that is, TCGA 183S; including $7 \mathrm{t}(8 ; 21), 11$ inv(16), $17 \mathrm{t}(15 ; 17), 9 \mathrm{MLL}$-rearranged, 3 $\mathrm{t}(9 ; 22), 22$ complex, 78 normal karyotype and 36 others), which were generated by use of Affymetrix Human Genome U133Plus2.0 GeneChips. Among the 183 AML cases, 177 (including $7 \mathrm{t}(8 ; 21), 11$ inv(16), $16 \mathrm{t}(15 ; 17), 9 \mathrm{MLL}$-rearranged, $3 \mathrm{t}(9 ; 22)$ 22 complex, 75 normal karyotype and 34 others) also have microRNA expression profiles as detected by IlluminaGA_miRNASeq platform, and the mRNA/miRNA profile data of the 177 AML cases were collectively referred to as TCGA_177S. One hundred ninety-four adult de novo AML cases (including $7 \mathrm{t}(8 ; 21), 11$ inv(16), 15 $\mathrm{t}(15 ; 17), 9 M L L$-rearranged, $3 \mathrm{t}(9 ; 22), 24$ complex, 91 normal karyotype and 34 others) with DNA methylation data as detected by Infinium HumanMethylation450 BeadChip were referred to as TCGA_194S. The mRNA/miRNA expression data and methylation data were downloaded from https://tcga-data.nci.nih.gov/tcga/dataAccessMatrix.htm? mode=ApplyFilter\& showMatrix=true\&diseaseType=LAML\& 12:28 PM 4/6/ 2016tumorNormal=TN\&tumorNormal=T\&tumorNormal=NT

Cell culture and transfection. These experiments were conducted as described previously ${ }^{17,23}$ with some modifications. THP-1, KOCL-48 and MV4;11 cells were grown in RPMI medium 1640 (Invitrogen, Carlsbad, CA) containing 10\% FBS, 1\% HEPES and $1 \%$ penicillin-streptomycin. MONOMAC- 6 cells were maintained in RPMI 1640 supplemented with 10\% FBS, 1\% HEPES, 2 mM L-glutamine, $100 \times$ Non-Essential Amino Acid, $1 \mathrm{mM}$ sodium pyruvate, $9 \mu \mathrm{g} \mathrm{ml}^{-1}$ insulin and $1 \%$ penicillin-streptomycin. Plasmids or siRNAs were transfected into MONOMAC-6 cells with Cell Line Nucleofector Kit V following program T-037, and THP-1 and KOCL-48 cells following program U-001, using the Amaxa Nucleofector Technology (Amaxa Biosystems, Berlin, Germany). Experiments were performed $48 \mathrm{~h}$ after transfection.

For the ATRA-treatment study, THP-1 cells were seeded at a concentration of $0.4 \times 10^{6} \mathrm{ml}^{-1}$ and treated with ATRA $\left(1 \mu \mathrm{moll}^{-1}\right)$ or vehicle control (DMSO, $0.001 \%$ ) for $72 \mathrm{~h}$ before cells were collected for RNA analysis or chromatin immunoprecipitation (ChIP) assays.

The MLL-ENL-ERtm cell line was kept in RPMI 1640 supplemented with interleukin 3 (IL-3), IL-6 and granulocyte-macrophage colony-stimulating factor (GM-CSF), $10 \mathrm{ng} \mathrm{ml}^{-1}$; stem cell factor (SCF) $100 \mathrm{ng} \mathrm{ml}^{-1} ; 10 \% \mathrm{FBS}$ and $1 \%$ penicillin-streptomycin. 4-OHT (Sigma-Aldrich, St Louis, MO) was added at a 100-nM final concentration as a 1-mM stock solution in ethanol. Cells were collected for experiments at the indicated days after drug withdrawal.

The MEF and HEK-293T cells were kept in DMEM (Invitrogen) containing $10 \%$ FBS, 1\% HEPES and 1\% penicillin-streptomycin; HEK-293T cells were transfected with Qiagen Effectene Transcription Kit (Qiagen) following the manufacturer's protocol.

All the cell lines were mycoplasma negative.

Lentivirus production and infection. All the plasmid for packaging lentivirus, including pMD2.G, pMDLg/pRRE and pRSV-Rev, were purchased from Addgene (Cambridge, MA). First, $0.5 \mu \mathrm{g}$ pMD2.G, $0.3 \mu \mathrm{g}$ pMDLg/pRRE, $0.7 \mu \mathrm{g}$ pRSV-Rev and $1.5 \mu \mathrm{g}$ shRNA constructs, that is shGFI1 and control (purchased from GE Dharmacon, Pittsburgh, PA) were co-transfected into HEK-293T cells in $60 \mathrm{~mm}$ cell culture dish with Effectene Transfection Reagent (Qiagen). The lentivirus particles were harvested at 48 and $72 \mathrm{~h}$ after transfection and concentrated with PEG-it Virus Precipitation Solution (SBI). Finally, the lentivirus particles were 
directly added into leukaemic cells and these cells were washed with PBS 24-48 h after infection.

Viability and proliferation assays. These experiments were conducted as described previously ${ }^{17,23}$ with some modifications. For apoptosis and viability assays, $48 \mathrm{~h}$ after transfection, cells were collected and seeded with requested concentration. Cell apoptosis and viability were assessed using ApoLive-Glo Multiplex Assay Kit (Promega, Madison, WI) following the corresponding manufacturer's manuals. For cell proliferation assays, per million cells were electroporated with $1.5 \mu \mathrm{g}$ plasmid. Twenty-four hours after transfection, cells were seeded in 96-well plates at the concentration of 10,000 cells per well. Cell numbers were counted at the indicating days.

Plasmid construction. The home-prepared expression vector of miR-22, that is MSCV-PIG-miR-22, was amplified by PCR using primers: forward: $5^{\prime}$-GCCCTCG AGTCTAGACTCCAGTTC- $3^{\prime}$ and reverse: $5^{\prime}$-GGGGAATTCCTACTCCTCAAT CCAG-3', and was subsequently cloned into the XhoI and EcoRI sites of the retrovirus vector MSCV-PIG (that is, MSCV-puro-IRES-GFP vector; bearing GFP gene), a kind gift from Drs Gregory Hannon, Scott Hammond and Lin He (Cold Spring Harbor Laboratory, Cold Spring Harbor, NY). The other miR-22 construct, the MSCV-PIG-miR-22 2 expression vector, was a gift from Dr Pier P. Pandolfi (Harvard Medical School, Boston, MA) ${ }^{59}$. The MSCVneo-MLL-AF9 plasmid $^{60}$ was kindly provided by Dr Scott Armstrong (Harvard Medical School, Boston, MA). The MSCV-FLT3-CDS plasmid ${ }^{61}$ was a gift from Dr Michael Cleary (Stanford University, Stanford, CA). The p1005-Crtc1 plasmid was provided by Dr Sheena Josselyn (The Hospital for Sick Children, Toronto, Canada) ${ }^{62}$, and sub-cloned into MSCV-PIG vector. The MSCV-PIG-MYCBP plasmid was PCR-amplified using primers: forward $5^{\prime}$-AAACTCGAGATGGCCCATTACAAAGC- $3^{\prime}$ and reverse $5^{\prime}$-CCGGAATTCCTATTCAGCACGC- $3^{\prime}$. The $3^{\prime}$ UTR constructs of CRTC1 and FLT3 containing putative binding sites for miR-22 were amplified by PCR from human normal bone marrow mononuclear cells using the primers below:

CRTC1-3'UTR: forward 5'-GCCATTACTAGTCCCACCTGAGTG-3' and reverse $5^{\prime}$-GCCATTAAGCTTGAGGACAGAAGC- $3^{\prime}$;

FLT3-3'UTR: forward 5'-GCCGCCACTAGTAGGAACAATTTAGTTTTAAG G- $3^{\prime}$ and reverse $5^{\prime}$-CGCAAGCTTGTGGGGACAAGAGTAACTTTA- $3^{\prime}$, and then cloned into pMIR-REPORT Luciferase miRNA Expression Reporter Vector (Ambion, Austin, TX). Site mutations were induced by PCR based on the sequence shown previously for the miR-22 binding site(s) mutant of $3^{\prime}$ UTR of CRTC1 and FLT3. The miR-22 promoter region $(-1,100 /+55 \mathrm{bp}$, as was identified by Bar et $a .^{45}$ ) was PCR-amplified using primers: forward $5^{\prime}$-AATAATGAGCTCAAGG TCGGACG- $3^{\prime}$ and reverse $5^{\prime}$-AATAATGATATCCTTTAGCTGGGTC- ${ }^{\prime}$, and cloned into the SacI and EcoRV sites of the pGL4.15 Luciferase Reporter Vector (Promega). The MSCV-Tet1 construct was as described previously ${ }^{14}$. All the above insertions were confirmed by DNA sequencing.

Chromatin immunoprecipitation. ChIP assay was performed, as described previously $^{14,17}$, with SABiosciences Corporation's ChampionChiP One-Day kit (Qiagen, Frederick, MD) following the manufacturer's protocol, with some modifications. Briefly, pellets of $5 \times 10^{6}$ cells were treated with fresh fixing buffer ( $1 \%$ formaldehyde) for $10 \mathrm{~min}$ at $37^{\circ} \mathrm{C}$ to crosslink DNA and proteins. The reaction was terminated by the addition of stop buffer and incubated at room temperature for $5 \mathrm{~min}$. After cell lysis, the cross-linked chromatin was sonicated to an average size of $\sim 500 \mathrm{bp}$ and was immunoprecipitated with antibodies against TET1, GFI1 (Santa Cruz Biotechnology Inc., Santa Cruz, CA), the $\mathrm{N}^{\prime}$-terminal portion of MLL (MLL-N), the $C^{\prime}$-terminal of MLL (MLL-C), H3K27Me3, H3K4Me3, RNA polymerase II, EZH2, SIN3A or IgG (Abcam, Cambridge, MA). Purified ChIP DNA was amplified by real-time qPCR using specific primers targeting the CpG-enriched upstream region of human miR-22: forward: $5^{\prime}$-GTT GTTGGAGTCGTGAGTG-3'; reverse: $5^{\prime}$-CGCTCCACCTTTCCTTAAA- $3^{\prime}$; or mouse miR-22: forward: $5^{\prime}$-TGAATGGGCGGGAGTAA-3'; reverse: $5^{\prime}$-CCACGA GCTGCGAATGAA-3'.

Bisulfite sequencing. THP-1 cells were treated with $1 \mu \mathrm{M}$ ATRA or DMSO control for $72 \mathrm{~h}$. Genomic DNA was extracted thereafter. One microgram of genomic DNA was then applied to MethylCode Bisulfite Conversion Kit (Invitrogen) following the manufacturers' instructions. After bisulfite conversion, $3 \mu \mathrm{l}$ of purified converted DNA was PCR-amplified using ZymoTaq DNA polymerase (Zymo Research, Irvine, CA) following the manufacturers' instructions. The PCR products were purified using PCR purification kits (Qiagen) and sent for sequencing. Primers applied in the PCR assays: miR-22 promoter: forward: $5^{\prime}$-TTT GTTTATTTTTGTTTTTTGGTT-3'; reverse: $5^{\prime}$-ACAACCCCTCCTTATTAAA ATC-3'; SLC43A2: forward: 5' -TGTTTTGTTTTTATGGAGTGATTTG-3'; reverse: $5^{\prime}$-AAAAATAACCATAAACCATCCTTCC-3'

Luciferase reporter and mutagenesis assays. Luciferase reporter and mutagenesis assays were conducted as described previously ${ }^{17,23}$, with some modifications. Briefly, for transfection, HEK-293T cells were plated in 96-well plates at a concentration of 6,000 cells per well in triplicate for each condition. For the miR-22 targeting CRTC1 and FLT3 experiments, after overnight incubation, cells were transfected with $20 \mathrm{ng}$ of the pMIR-REPORT bearing the CRTC1 or FLT3 $3^{\prime}$ UTR or the $3^{\prime}$ UTRs with miR-22 binding site mutations, and $20 \mathrm{ng}$ of MSCV-miR-22 or an empty MSCV vector using Effectene Transfection Reagent (Qiagen) according to the manufacturer's protocol. pMIR-REPORT Betagalactosidase Reporter Control Vector (Ambion) (1 ng) was co-transfected for transfection efficiency control in all transfections. Cells were lysed and firefly luciferase and $\beta$-galactosidase activities were detected using Dual-Light Combined Reporter Gene Assay System (Applied Biosystems, Foster City, CA) 48 h post transfection. Firefly luciferase activity was normalized to $\beta$-galactosidase activity for each transfected well. For the Tet1 targeting miR-22 study, HEK-293T cells were transfected with $20 \mathrm{ng}$ MSCV-Tet1 construct and/or 20 ng pGL4.15-miR-22 promoter. The succeeding luciferase reporter assay was conducted according to the manufacture's protocol (Promega). Each experiment was performed in triplicate and repeated three times.

Co-immunoprecipitation analysis. For immunoprecipitation, cells were washed with ice-cold PBS and lysed in $800 \mu$ l Nonidet P-40 solubilization buffer (50 mM Hepes, pH 8.0, $250 \mathrm{mM} \mathrm{NaCl}, 0.5 \%$ Nonidet P-40, $10 \%$ glycerol, $2 \mathrm{mM}$ EDTA, $1 \mathrm{mM} \mathrm{NaF}$, plus $10 \mu \mathrm{g} \mathrm{ml}^{-1}$ aprotinin, $10 \mu \mathrm{g} \mathrm{ml}^{-1}$ benzamidine and $0.2 \mathrm{mM}$ PMSF). The following procedures are performed as described previously ${ }^{63}$ GFI1 was precipitated by using protein A Sepharose beads coated with $400 \mathrm{ng}$ rabbit anti-GFI1 antibody (Santa Cruz Biotechnology Inc.). Lysates and immunoprecipitation complexes were separated and detected by western blotting

\section{Packaging of recombinant retroviruses and CFA assays. Those experiments} were conducted as described previously ${ }^{17,23}$ with some modifications. Briefly, retrovirus vectors were co-transfected with pCL-Eco packaging vector (IMGENEX, San Diego, CA) into HEK-293T cells using Effectene Transfection Reagent (Qiagen) to produce the retroviruses. BM cells were collected from a cohort of 4-6-week-old B6.SJL (CD45.1) donor mice after 5 days of 5-fluorouracil (5-FU) treatment, and primitive hematopoietic progenitor cells were enriched with Mouse Lineage Cell Depletion Kit (Miltenyi Biotec Inc., Auburn, CA). An aliquot of enriched hematopoietic progenitor cells were added to retroviral supernatant together with polybrene in a conical tube, which were centrifuged at $2,000 \mathrm{~g}$ for $2 \mathrm{~h}$ at $32^{\circ} \mathrm{C}$ (that is, 'spinoculation, $14,17,23$ ) and then the media was replaced with fresh media and incubated for $20 \mathrm{~h}$ at $37^{\circ} \mathrm{C}$. Next day, the same procedure was repeated once.

Then, on the day following the second spinoculation, an equivalent of $2.0 \times 10^{4}$ cells were plated into a 35-mm Petri dish in $1.5 \mathrm{ml}$ of Methocult M3230 methylcellulose medium (Stem Cell Technologies Inc., Vancouver, Canada) containing $10 \mathrm{ng} \mathrm{ml}^{-1}$ each of murine recombinant IL-3, IL-6, and granulocytemacrophage colony-stimulating factor (GM-CSF), and $30 \mathrm{ng} \mathrm{ml}^{-1}$ of murine recombinant SCF (R\&D Systems, Minneapolis, MN), along with $1.0 \mathrm{mg} \mathrm{ml}^{-1}$ of G418 and/or $2 \mu \mathrm{g} \mathrm{ml}^{-1}$ of puromycin. For each transduction, there were two duplicate dishes. Cultures were incubated at $37^{\circ} \mathrm{C}$ in a humidified atmosphere of $5 \% \mathrm{CO}_{2}$ in air. The colonies were replated every 7 days under the same conditions. The colony-forming/replating assays were repeated three times.

Primary and secondary BMT. These experiments were conducted as described previously ${ }^{14,17,23}$ with some modifications.

For primary BMT assays shown in Fig. 1e, normal bone marrow cells of B6.SJL (CD45.1) mice were retrovirally transduced with MSCV-neo + MSCV-PIG (as control; Ctrl), MSCV-neo + MSCV-PIG-miR-22 (that is, miR-22), MSCV-neo$M L L-A F 9+$ MSCV-PIG (that is, MA9), MSCV-neo-MLL-AF9 + MSCV-PIG-miR22 (that is, MA9 + miR-22) or MSCV-neo- $M L L-A F 9+$ MSCV-PIG-miR-22 mutant (that is, MA9 + miR-22mut), through two rounds of spinoculation. Then, retrovirally transduced cells were plated into methylcellulose medium supplied with a set of cytokines to form colonies as described in the CFA assays. Seven days later, colony cells were collected and washed, and then were injected by tail vein into lethally irradiated (960 rads) 8-10-week-old C57BL/6 (CD45.2) recipient mice with $1.5 \times 10^{5}$ donor cells plus a radioprotective dose of whole BM cells $\left(1 \times 10^{6}\right.$; freshly collected from a C57BL/6 mouse) per recipient mouse. Notably, as the colony cells were under selection of both G418 $\left(1.0 \mathrm{mg} \mathrm{ml}^{-1}\right)$ and puromycin $\left(2 \mu \mathrm{g} \mathrm{ml}^{-1}\right)$ for a week, all donor cells (that is, the collected colony cells) must be positive for retroviral transductions of both MSCVneo- and MSCV-PIG-based constructs. Thus, $M L L-A F 9$ and miR-22 (or miR-22mut) must be ectopically co-expressed in MA9 + miR-22 (or MA9 + miR-22mut) donor cells, which actually were confirmed by qPCR. Indeed, due to the potent inhibitory effect of miR-22 on MLL-AF9-induced colony forming, we had to prepare more mouse BM progenitor cells for the co-transduction of MLL-AF9 and miR-22. Thus, we plated them in a larger number of dishes than what we did for other groups of co-transductions. After BMT, all recipient mice were watched for leukemogenesis for a period of 200 days or till the end point that the mice developed full-blown AML or other severe illness.

For primary BMT assays shown in Fig. 1g, C57BL/6 mouse (CD45.2) BM progenitor cells were co-transduced with MSCVneo-MLL-AF10, together with MSCV-PIG-miR-22 or MSCV-PIG vector. Cells were grown in RPMI medium 1640 (Invitrogen) containing $10 \%$ FBS, $1 \%$ HEPES and $1 \%$ penicillin- 
streptomycin, supplemented with a supply of SCF, IL-3 and IL-8. The cells were selected with both puromycin and G418 for 7 days before transplantation. After that, $1.5 \times 10^{5}$ donor cells plus a radioprotective dose of whole bone marrow cells $\left(1 \times 10^{6}\right.$; freshly collected from a B6.SJL (CD45.1) mouse) were injected into per lethally irradiated (960 rads) 8-10-week-old B6.SJL recipient mouse.

For primary BMT assays shown in Fig. 1h, C57BL/6 mouse (CD45.2) $\mathrm{BM}$ progenitor cells or miR-22-1- $\mathrm{BM}$ progenitor cells were retrovirally transduced with MSCV-PIG-AE9a. The cells were selected with puromycin for 7 days before transplantation. After that, $1.5 \times 10^{5}$ donor cells plus a radioprotective dose of whole bone marrow cells $\left(1 \times 10^{6}\right.$; freshly collected from a B6.SJL (CD45.1) mouse) were injected into per lethally irradiated (960 rads) 8-10-week-old B6.SJL recipient mouse.

For secondary BMT assay shown in Fig. 2a,c,d, leukaemic BM cells isolated from the primary leukaemic mice bearing MLL-AF9, AML1-ET9a (AE9a) or FLT3$\mathrm{ITD} / \mathrm{NPMIc}{ }^{+}$were retrovirally transduced with MSCV-PIG + MSCVneo (as control; MA9-AML + Ctrl, AE9a-AML + Ctrl or FLT3-ITD/NPM1c ${ }^{+}$$\mathrm{AML}+\mathrm{Ctrl}$ ) or MSCV-PIG + MSCVneo-miR-22 (that is, MA9-AML + miR-22, AE9a-AML + miR-22, or FLT3-ITD/NPM1c ${ }^{+}$-AML + miR-22). Similarly, retrovirally transduced cells were plated into methylcellulose medium supplied with puromycin and G418 (for selection) and a set of cytokines to form colonies. Seven days later, the colony cells were collected and washed, and then were transplanted into sub-lethally irradiated (480 rads) 8 -10-week-old C57BL/6 (CD45.2) secondary recipient mice via tail vein injection, with the dosage of $1.5 \times 10^{5}$ donor cells per recipient mouse.

For secondary BMT assays shown in Fig. 4f, leukaemic BM cells isolated from the primary leukaemic mice bearing $M L L-A F 9$ fusion were retrovirally transduced with MSCV-neo + MSCV-PIG (that is, MA9-AML + Ctrl), MSCV-neo-miR-22 + MSCV-PIG (that is, MA9-AML + miR-22), MSCV-neo-miR-22 + MSCV-PIGCRTC1 (that is, MA9-AML + miR-22 + CRTC1), MSCV-neo-miR-22 + MSCVPIG-FLT3 (that is, MA9-AML + miR-22 + FLT3) or MSCV-neo-miR-22 + MSCVPIG-MYCBP (that is, MA9-AML + miR-22 + MYCBP). Again, retrovirally transduced cells were plated into methylcellulose medium supplied with G418 and puromycin (for selection) as well as a set of cytokines to form colonies. Seven days later, the colony cells were collected and washed, and then were transplanted into sub-lethally irradiated (480 rads) 8-10-week-old C57BL/6 (CD45.2) secondary recipient mice via tail vein injection, with the dosage of $1.5 \times 10^{5}$ donor cells per recipient mouse.

\section{Preparation of Cy5.5-labelled G7 PAMAM dendrimers. G7 PAMAM} dendrimers obtained from Sigma-Aldrich were purified and fluorescently labelled using an $\mathrm{N}$-hydroxysuccinimide ester of cyanine5.5 (NHS-Cy5.5) (Lumiprobe Corporation, Hallandale Beach, FL), as has been previously reported ${ }^{64}$. In brief, G7 PAMAM dendrimers $\left(38.7 \mathrm{mg}, 332 \mathrm{nmol}\right.$ ) were dissolved in $2 \mathrm{ml} \mathrm{ddH}_{2} \mathrm{O}$, to which NHS-Cy5.5 (3.75 mg, $3.32 \mu \mathrm{mol})$ in $400 \mu \mathrm{l}$ DMSO was added dropwise, and the reaction allowed to proceed under vigorous stirring for $24 \mathrm{~h}$ at room temperature. Excess NHS-Cy5.5 was removed using an Amicon Ultra-15 Centrifugal Filter Unit (MWCO 10,000, Millipore, Billerica, MA) at 4,000 r.p.m. and $4{ }^{\circ} \mathrm{C}$ for $20 \mathrm{~min}$ and washing with $\mathrm{ddH}_{2} \mathrm{O} 10$ times. Remaining product was re-dissolved in $\mathrm{ddH}_{2} \mathrm{O}$ and lyophilized, resulting in G7-Cy5.5- $\mathrm{NH}_{2}$. All products were characterized by ${ }^{1} \mathrm{H}$ NMR using a 400-MHz Bruker DPX-400 spectrometer (Bruker BioSpin Corp., Billerica, MA).

Nanoparticle treatment in BMT or xeno-transplantation models. For secondary BMT followed with G7-Cy5.5- $\mathrm{NH}_{2}$ dendrimer treatment shown in Fig. 7a,b, leukaemic BM cells isolated from the primary leukaemic mice (CD45.1) bearing $M L L-A F 9$ or $A E 9 a$ fusion were transplanted into sub-lethally irradiated (480 rads) 8-10-week-old C57BL/6 (CD45.2) secondary recipient mice via tail vein injection, with the dosage of $1.5 \times 10^{5}$ donor cells per recipient mouse. After the onset of leukaemia (when mice had an engraftment (CD45.1) over $20 \%$ and/or white blood cell counts higher than $4 \times 10^{9} 1^{-1}$; for the MLL-AF9 and AE9a secondary transplantation models, usually 10 days post transplantation), the recipient mice were injected with PBS control, or $0.5 \mathrm{mg} \mathrm{kg}^{-1} \mathrm{miR}-22$ or miR-22 mutant RNA oligos formulated with G7-NH2 nanoparticles, i.v., every other day, until the PBStreated group all died of leukaemia.

For xeno-transplantation followed with G7-Cy5.5-NH2 dendrimer treatment shown in Fig. 7c, MV4;11 cells were transplanted into NSGS (NSG-SGM3) mice via tail vein injection, with the dosage of $5 \times 10^{5}$ donor cells per recipient mouse. Five days after xeno-BMT, the recipient mice were injected with PBS control, or $0.5 \mathrm{mg} \mathrm{kg}^{-1}$ miR-22 or miR-22 mutant RNA oligos formulated with G7-NH2nanoparticles, i.v., every other day, until the PBS-treated mice all died of leukaemia.

The maintenance and monitoring of mice. C57BL/6 (CD45.2), B6.SJL (CD45.1) mice were purchased from the Jackson Lab (Bar Harbor, ME, USA) or Harlan Laboratories, Inc (Indianapolis, IN, USA). NSGS (NSG-SGM3) immunodeficient mice ${ }^{49}$ and miR-22 $-1-$ (ref. 20) mice were purchased from the Jackson Lab and were bred and maintained in house. Both male and female mice were used for the experiments. All laboratory mice were maintained in the animal facility at the University of Chicago and the University of Cincinnati. All experiments on mice in our research protocol were approved by Institutional Animal Care and Use Committee (IACUC) of the University of Chicago and the University of
Cincinnati. The maintenance, monitoring and end-point treatment of mice were conducted as described previously $14,17,23$

Western blotting. Western blotting was conducted as described previously $14,17,23$ with some modifications. Briefly, transiently transfected MONOMAC-6 cells were collected and lysed with RIPA buffer (Thermo Scientific, BufferRockford, IL). Proteins from the lysate were fractionated by electrophoresis through $4-15 \%$ polyacrylamide gels (Bio-rad, Hercules, CA) and transferred to polyvinylidene fluoride membranes using tris-glycine transfer buffer (Thermo Scientific). Blots were incubated with IRDye $800 \mathrm{CW}$-conjugated or $700 \mathrm{CW}$-conjugated antibody and infrared fluorescence images were obtained with the Odyssey infrared imaging system (Li-Cor Bioscience, Lincoln, NE). 100-200 $\mathrm{ng} \mathrm{ml}^{-1}$ anti-CRTC1, anti-FLT3, anti-MYCBP, anti-BMI1, anti-CDK6, anti-PGK1 (Santa Cruz Biotechnology Inc.) anti-HMGA1 (Abcam) and anti-GAPDH (Thermo Scientific) antibodies were used to detect corresponding proteins. Original scans can be found as Supplementary Figures 7-9.

Target gene prediction. Putative targets of miR-22 was predicted by TargetScan (http://www.targetscan.org) ${ }^{18}$

DNA copy-number analysis of miR-22 gene locus in human AML. The copynumber data of AML from The Cancer Genome Atlas (TCGA) project were downloaded from Broad Firehose's analyses runs. The putative copy-number calls were determined using GISTIC 2.0 (ref. 65). The latest GISTIC analyses data were obtained using the following shell command: 'firehose_get -o 'GISTIC' analyses latest LAML'

The.cel files of Affymetrix SNP 6.0 data for GSE21107 (ref. 66) and GSE23452 (ref. 67) were downloaded from NCBI GEO. The raw data were preprocessed using PennCNV ${ }^{68}$. Then ASCAT ${ }^{69}$ was used to obtain the copy-number alterations. The putative copy-number calls were determined using GISTIC 2.0 as described above.

Software and statistical analyses. The miRNA and gene/exon array data analyses, as well as qPCR data analyses were conducted by use of Partek Genomics Suite (Partek Inc.), TIGR Mutiple Array Viewer software package (TMeV version 4.6; TIGR, Rockville, MA) ${ }^{70}$ and/or Bioconductor R packages. The miRNA-gene expression correlation was analysed by use of Partek Genomics Suite (Partek Inc.) The $t$-test, Kaplan-Meier method and log-rank testand so on were performed with WinSTAT (R. Fitch Software), GraphPad Prism version 5.00 (GraphPad Software, San Diego, CA) and/or Partek Genomics Suite (Partek Inc.). The $P$ values $<0.05$ were considered as statistically significant. Significance analysis of microarrays, embedded in the TMeV package (TIGR, Rockville, MA), was used to identify the genes that are significantly $(q<0.05$; false discovery rate, FDR $<0.05)$ dysregulated in the MLL-AF9-mediated mouse leukaemia samples or human AML samples relative to the normal controls. Pearson correlation was used in the analysis of the correlation between miR-22 and candidate genes in expression. The list of transcription factors that have evolutionarily conserved binding sites within the miR-22 promoter region (that is, the adjacent upstream CpG island) was obtained by searching UCSC Genome Browser (https://genome.ucsc.edu/cgi-bin/ hgTracks?db=hg19\&position=chr17\%3A1614689-1623188\&hgsid= 467686877 3vyTlry3a40ZiT7dfAaAIAsYA2R6).

Data availability. Data referenced in this study are available in The Gene Expression Omnibus. The Affymetrix exon array data and the microarray data are available under accession codes GSE34184 and GSE30285. Additional exon array data are available under accession code GSE27370. The mouse microarray data is available under accession code GSE34185. The AML samples collected by the German AMLCG study group are available under accession code GSE37642. The mRNA gene expression data of 183 adult de novo AML cases is available from the TCGA.

\section{References}

1. Zeisig, B. B., Kulasekararaj, A. G., Mufti, G. J. \& So, C. W. SnapShot: acute myeloid leukemia. Cancer Cell 22, 698-698.e1 (2012).

2. Estey, E. \& Dohner, H. Acute myeloid leukaemia. Lancet 368, 1894-1907 (2006)

3. Grimwade, D. \& Mrozek, K. Diagnostic and prognostic value of cytogenetics in acute myeloid leukemia. Hematol. Oncol. Clin. North Am. 25, 1135-1161 vii (2011)

4. Chen, J., Odenike, O. \& Rowley, J. D. Leukaemogenesis: more than mutant genes. Nat. Rev. Cancer 10, 23-36 (2010).

5. Graubert, T. \& Walter, M. J. Genetics of myelodysplastic syndromes: new insights. Hematology Am. Soc. Hematol. Educ. Program 2011, 543-549 (2011)

6. Figueroa, M. E. et al. MDS and secondary AML display unique patterns and abundance of aberrant DNA methylation. Blood 114, 3448-3458 (2009).

7. Griffiths, E. A. \& Gore, S. D. Epigenetic therapies in MDS and AML. Adv. Exp. Med. Biol. 754, 253-283 (2013).

8. Wu, H. et al. Dual functions of Tetl in transcriptional regulation in mouse embryonic stem cells. Nature 473, 389-393 (2011).

9. Williams, K. et al. TET1 and hydroxymethylcytosine in transcription and DNA methylation fidelity. Nature 473, 343-348 (2011). 
10. $\mathrm{Hu}, \mathrm{X}$. et al. Tet and TDG mediate DNA demethylation essential for mesenchymal-to-epithelial transition in somatic cell reprogramming. Cell Stem Cell 14, 512-522 (2014).

11. Delhommeau, F. et al. Mutation in TET2 in myeloid cancers. N. Engl. J. Med. 360, 2289-2301 (2009).

12. Ko, M. et al. Impaired hydroxylation of 5-methylcytosine in myeloid cancers with mutant TET2. Nature 468, 839-843 (2010).

13. Moran-Crusio, K. et al. Tet2 loss leads to increased hematopoietic stem cell self-renewal and myeloid transformation. Cancer Cell 20, 11-24 (2011).

14. Huang, H. et al. TET1 plays an essential oncogenic role in MLL-rearranged leukemia. Proc. Natl Acad. Sci. USA 110, 11994-11999 (2013).

15. Song, S. J. et al. MicroRNA-antagonism regulates breast cancer stemness and metastasis via TET-family-dependent chromatin remodeling. Cell 154, 311-324 (2013).

16. Song, S. J. et al. The oncogenic microRNA miR-22 targets the TET2 tumor suppressor to promote hematopoietic stem cell self-renewal and transformation. Cell Stem Cell 13, 87-101 (2013).

17. Jiang, X. et al. Blockade of miR-150 maturation by MLL-fusion/MYC/LIN-28 is required for MLL-associated leukemia. Cancer Cell 22, 524-535 (2012).

18. Lewis, B. P., Burge, C. B. \& Bartel, D. P. Conserved seed pairing, often flanked by adenosines, indicates that thousands of human genes are microRNA targets. Cell 120, 15-20 (2005).

19. Yan, M. et al. A previously unidentified alternatively spliced isoform of $t(8 ; 21)$ transcript promotes leukemogenesis. Nat. Med. 12, 945-949 (2006).

20. Gurha, P. et al. Targeted deletion of microRNA-22 promotes stress-induced cardiac dilation and contractile dysfunction. Circulation 125, 2751-2761 (2012).

21. He, C., Li, Z., Chen, P., Huang, H., Hurst, L. D. \& Chen, J. Young intragenic miRNAs are less coexpressed with host genes than old ones: implications of miRNA-host gene coevolution. Nucleic Acids Res. 40, 4002-4012 (2012).

22. Ley, T. J. et al. Genomic and epigenomic landscapes of adult de novo acute myeloid leukemia. N. Engl. J. Med. 368, 2059-2074 (2013).

23. Li, Z. et al. miR-196b directly targets both HOXA9/MEIS1 oncogenes and FAS tumour suppressor in MLL-rearranged leukaemia. Nat. Commun. 2, 688 (2012).

24. Wang, Z. et al. GSK-3 promotes conditional association of CREB and its coactivators with MEIS1 to facilitate HOX-mediated transcription and oncogenesis. Cancer Cell 17, 597-608 (2010).

25. Cheng, J. C. et al. CREB is a critical regulator of normal hematopoiesis and leukemogenesis. Blood 111, 1182-1192 (2008).

26. Sandoval, S., Pigazzi, M. \& Sakamoto, K. M. CREB: a key regulator of normal and neoplastic hematopoiesis. Adv. Hematol. 2009, 634292 (2009).

27. Haferlach, C. et al. ETV6 rearrangements are recurrent in myeloid malignancies and are frequently associated with other genetic events. Genes Chromosomes Cancer 51, 328-337 (2012).

28. Armstrong, S. A. et al. Inhibition of FLT3 in MLL. Validation of a therapeutic target identified by gene expression based classification. Cancer Cell 3, 173-183 (2003).

29. Takahashi, S. Downstream molecular pathways of FLT3 in the pathogenesis of acute myeloid leukemia: biology and therapeutic implications. J. Hematol. Oncol. 4, 13 (2011).

30. Xiong, J., Du, Q. \& Liang, Z. Tumor-suppressive microRNA-22 inhibits the transcription of E-box-containing c-Myc target genes by silencing c-Myc binding protein. Oncogene 29, 4980-4988 (2010).

31. Zeisig, B. B. et al. Hoxa9 and Meis1 are key targets for MLL-ENL-mediated cellular immortalization. Mol. Cell Biol. 24, 617-628 (2004).

32. Pigazzi, M. et al. MicroRNA-34b promoter hypermethylation induces CREB overexpression and contributes to myeloid transformation. Haematologica 98, 602-610 (2013).

33. Placke, T. et al. Requirement for CDK6 in MLL-rearranged acute myeloid leukemia. Blood 124, 13-23 (2014).

34. Ayton, P. M. \& Cleary, M. L. Transformation of myeloid progenitors by MLL oncoproteins is dependent on Hoxa7 and Hoxa9. Genes Dev. 17, 2298-2307 (2003).

35. Schwable, J. et al. RGS2 is an important target gene of Flt3-ITD mutations in AML and functions in myeloid differentiation and leukemic transformation. Blood 105, 2107-2114 (2005).

36. Smith, L. L. et al. Functional crosstalk between Bmi1 and MLL/Hoxa9 axis in establishment of normal hematopoietic and leukemic stem cells. Cell Stem Cell 8, 649-662 (2011).

37. Wu, X., Qin, L., Fako, V. \& Zhang, J. T. Molecular mechanisms of fatty acid synthase (FASN)-mediated resistance to anti-cancer treatments. Adv. Biol. Regul. 54, 214-221 (2014).

38. Xu, Y. et al. The HMG-I oncogene causes highly penetrant, aggressive lymphoid malignancy in transgenic mice and is overexpressed in human leukemia. Cancer Res. 64, 3371-3375 (2004)

39. Jacoby, M. A. \& Walter, M. J. Detection of copy number alterations in acute myeloid leukemia and myelodysplastic syndromes. Expert Rev. Mol. Diagn. 12, 253-264 (2012).

40. Ninomiya, S. et al. Integrated analysis of gene copy number, copy neutral LOH, and microRNA profiles in adult acute lymphoblastic leukemia. Cytogenet. Genome Res. 136, 246-255 (2012).
41. Konishi, H. et al. Detailed characterization of a homozygously deleted region corresponding to a candidate tumor suppressor locus at distal $17 \mathrm{p} 13.3$ in human lung cancer. Oncogene 22, 1892-1905 (2003).

42. Sankar, M. et al. Identification of a commonly deleted region at $17 \mathrm{p} 13.3$ in leukemia and lymphoma associated with $17 \mathrm{p}$ abnormality. Leukemia 12, 510-516 (1998).

43. Chattopadhyay, P. et al. Loss of heterozygosity of a locus on 17p13.3, independent of $\mathrm{p} 53$, is associated with higher grades of astrocytic tumours. Oncogene 15, 871-874 (1997).

44. Li, Z. et al. Identification of a 24-gene prognostic signature that improves the European LeukemiaNet risk classification of acute myeloid leukemia: an international collaborative study. J. Clin. Oncol. 31, 1172-1181 (2013).

45. Bar, N. \& Dikstein, R. miR-22 forms a regulatory loop in PTEN/AKT pathway and modulates signaling kinetics. PLoS ONE 5, e10859 (2010).

46. Drach, J., Lopez-Berestein, G., McQueen, T., Andreeff, M. \& Mehta, K. Induction of differentiation in myeloid leukemia cell lines and acute promyelocytic leukemia cells by liposomal all-trans-retinoic acid. Cancer Res. 53, 2100-2104 (1993).

47. Duan, Z. \& Horwitz, M. Targets of the transcriptional repressor oncoprotein Gfi-1. Proc. Natl Acad. Sci. USA 100, 5932-5937 (2003).

48. Pack, D. W., Hoffman, A. S., Pun, S. \& Stayton, P. S. Design and development of polymers for gene delivery. Nat. Rev. Drug Discov. 4, 581-593 (2005).

49. Wunderlich, M. et al. AML xenograft efficiency is significantly improved in NOD/SCID-IL2RG mice constitutively expressing human SCF, GM-CSF and IL-3. Leukemia 24, 1785-1788 (2010).

50. Cartron, P. F., Nadaradjane, A., Lepape, F., Lalier, L., Gardie, B. \& Vallette, F. M. Identification of TET1 partners that control its DNA-demethylating function. Genes Cancer 4, 235-241 (2013).

51. Saleque, S., Kim, J., Rooke, H. M. \& Orkin, S. H. Epigenetic regulation of hematopoietic differentiation by Gfi-1 and Gfi-1b is mediated by the cofactors CoREST and LSD1. Mol. Cell 27, 562-572 (2007).

52. Thambyrajah, R. et al. GFI1 proteins orchestrate the emergence of haematopoietic stem cells through recruitment of LSD1. Nat. Cell Biol. 18, 21-32 (2016).

53. Abdel-Wahab, O. et al. Genetic characterization of TET1, TET2, and TET3 alterations in myeloid malignancies. Blood 114, 144-147 (2009).

54. Zuber, J. et al. RNAi screen identifies Brd4 as a therapeutic target in acute myeloid leukaemia. Nature 478, 524-528 (2011).

55. Huang, M. J., Cheng, Y. C., Liu, C. R., Lin, S. \& Liu, H. E. A small-molecule c-Myc inhibitor, 10058-F4, induces cell-cycle arrest, apoptosis, and myeloid differentiation of human acute myeloid leukemia. Exp. Hematol. 34, 1480-1489 (2006).

56. Ling, H., Fabbri, M. \& Calin, G. A. MicroRNAs and other non-coding RNAs as targets for anticancer drug development. Nat. Rev. Drug Discov. 12, 847-865 (2013).

57. Li, Z. et al. Distinct microRNA expression profiles in acute myeloid leukemia with common translocations. Proc. Natl Acad. Sci. USA 105, 15535-15540 (2008).

58. Irizarry, R. A. et al. Summaries of Affymetrix GeneChip probe level data. Nucleic Acids Res. 31, e15 (2003).

59. Poliseno, L. et al. Identification of the miR-106b $\sim 25$ microRNA cluster as a proto-oncogenic PTEN-targeting intron that cooperates with its host gene MCM7 in ransformation. Sci. Signal. 3, ra29 (2010).

60. Krivtsov, A. V. et al. Transformation from committed progenitor to leukaemia stem cell initiated by MLL-AF9. Nature 442, 818-822 (2006).

61. Somervaille, T. C. et al. Hierarchical maintenance of MLL myeloid leukemia stem cells employs a transcriptional program shared with embryonic rather than adult stem cells. Cell Stem Cell 4, 129-140 (2009).

62. Sekeres, M. J. et al. Increasing CRTC1 function in the dentate gyrus during memory formation or reactivation increases memory strength without compromising memory quality. J. Neurosci. 32, 17857-17868 (2012).

63. Jiang, X., Yang, P. \& Ma, L. Kinase activity-independent regulation of cyclin pathway by GRK2 is essential for zebrafish early development. Proc. Natl Acad. Sci. USA 106, 10183-10188 (2009).

64. Modi, D. A. et al. Targeting of follicle stimulating hormone peptide-conjugated dendrimers to ovarian cancer cells. Nanoscale 6, 2812-2820 (2014).

65. Mermel, C. H. et al. GISTIC2.0 facilitates sensitive and confident localization of the targets of focal somatic copy-number alteration in human cancers. Genome Biol. 12, R41 (2011).

66. Parkin, B. et al. NF1 inactivation in adult acute myelogenous leukemia. Clin. Cancer Res. 16, 4135-4147 (2010).

67. Parkin, B. et al. Acquired genomic copy number aberrations and survival in adult acute myelogenous leukemia. Blood 116, 4958-4967 (2010).

68. Wang, K. et al. PennCNV: an integrated hidden Markov model designed for high-resolution copy number variation detection in whole-genome SNP genotyping data. Genome Res. 17, 1665-1674 (2007).

69. Van Loo, P. et al. Allele-specific copy number analysis of tumors. Proc. Natl Acad. Sci. USA 107, 16910-16915 (2010).

70. Saeed, A. I. et al. TM4 microarray software suite. Methods Enzymol. 411, 134-193 (2006). 


\section{Acknowledgements}

Special thanks to the late Dr Janet Rowley for her long-term support. We also thank Drs Pier P. Pandolfi, Sheena Josselyn, Dong-Er Zhang, Scott Armstrong, Michael Cleary, James Mulloy, Robert Slany, Lin He and Michael Thirman for providing mouse models, retroviral constructs or cell lines, and the German AMLCG study group for AML microarray data. This work was supported by Leukemia \& Lymphoma Society (LLS) Translational Research Grant (J.C.), the National Institutes of Health (NIH) R01 Grants CA178454, CA182528 and CA127277 (J.C.), American Cancer Society (ACS) Research Scholar grant (J.C.), The University of Chicago Committee on Cancer Biology (CCB) Fellowship Program (X.J.), LLS Special Fellowship (Z.L.), Gabrielle's Angel Foundation for Cancer Research (J.C., Z.L., X.J. and H.H.) and Intramural Research Program of National Human Genome Research Institute, NIH (A.G.E. and P.P.L.). C.H. is an investigator of the Howard Hughes Medical Institute.

\section{Author contributions}

X.J. and J.C. conceived the project and designed the research. X.J., S.A., J.B., M.Y., Z.Z., P.C., H.H., B.U., C.H., S.G., H.W., Y.W., Y.L., J.S., S.L., A.G.E., Y.Y., J.S., M.B.N., Z.L. and J.C. performed experiments and/or data analyses; X.J., H.H., A.G.E., R.A.L., M.M.L.L., T.H., S.K.B., P.P.L., J.J., J.Z., Z.L., C.H., S.H. and J.C. contributed reagents/analytic tools and/or grant support; X.J. and J.C. wrote the paper. All authors discussed the results and commented on the manuscript.

\section{Additional information}

Accession codes: The microarray data have been deposited in the Gene Expression Omnibus (GEO) database under the accession code GSE34184, GSE30285, GSE27370, GSE34185, and GSE37642.

Supplementary Information accompanies this paper at http://www.nature.com/ naturecommunications

Competing financial interests: The authors declare no competing financial interests.

Reprints and permission information is available online at http://npg.nature.com/ reprintsandpermissions/

How to cite this article: Jiang, X. et al. miR-22 has a potent anti-tumour role with therapeutic potential in acute myeloid leukaemia. Nat. Commun. 7:11452 doi: 10.1038/ ncomms11452 (2016).

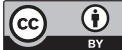

This work is licensed under a Creative Commons Attribution 4.0 International License. The images or other third party material in this article are included in the article's Creative Commons license, unless indicated otherwise in the credit line; if the material is not included under the Creative Commons license, users will need to obtain permission from the license holder to reproduce the material. To view a copy of this license, visit http://creativecommons.org/licenses/by/4.0/ 